\title{
Design of Surface Micromachined Compliant MEMS
}

\author{
by \\ Joe Anthony Bradley \\ A thesis submitted to the graduate faculty \\ in partial fulfillment of the requirement for the degree of \\ MASTER OF SCIENCE
}

Major: Mechanical Engineering

Program of Study Committee:

Radha Sarma, Co-major Professor

James Bernard, Co-major Professor

Gary Tuttle

lowa State University

Ames, lowa

2001 


\section{Graduate College lowa State University}

This is to certify that the Master's thesis of

Joe Anthony Bradley

has met the thesis requirement of the lowa State University

Committee Member

Co-major Professor

Co-major Professor

For the Major Program 


\section{TABLE OF CONTENTS}

LIST OF FIGURES $\quad$ V

LIST OF TABLES Vii

$\begin{array}{lll}\text { ABSTRACT } & \text { viii }\end{array}$

CHAPTER 1 - INTRODUCTION

1.1 Definition of MEMS 1

1.2 Problems with MEMS 2

1.3 Compliant Devices $\quad 2$

1.4 Research Outcomes $\quad 4$

1.5 Chapter Organization $\quad 5$

CHAPTER 2 - LITERATURE REVIEW

2.1 MEMS: Foundation and Fundamentals $\quad 6$

2.2 Compliant Mechanisms Design: Foundation and Overview 7

2.3 Topological Synthesis and Development of Compliant Mechanisms $\quad 10$

2.4 MEMS as Compliant Mechanisms 12

2.4.1 Related MEMS Designs 13

2.5 Microfabrication Process: Bulk and Surface Micromachining 15

2.6 The Future of MEMS Technologies and Applications 18

CHAPTER 3 - METHODOLOGY AND PROCEDURES

3.1 Geometrical Design 21

3.2 Problem Formulation 23

3.3 Determination of Design Requirements 24

3.4 Surface Micromachining Procedures in MEMS Fabrication 25

3.4.1 Mask Development and Design 25

3.4.2 Fabrication Processes and Technique 26

3.4.3 Material Properties 28

3.5 Design and Fabrication For The Compliant Micro-gripper and Micro-clamp 28

3.5.1 Design Methodology For The Compliant Micro-gripper and Micro-clamp 29

3.5.2 Fabrication Process For The Compliant Micro-gripper and Micro-clamp 31

CHAPTER 4 - RESULTS AND ANALYSIS

4.1 Description of Final Micro-Gripper and Micro-Clamp 35

4.2 Solution Technique $\quad 35$

4.2.1 Performance Criteria For Compliant Mechanism 36

4.2.2 Computation of Mechanical and Geometric Advantage 36

4.2.3 Analysis of Stress in Thin-Film 38

4.2.4 Manufacturability of the Devices $\quad 40$

4.3 FEA Analysis Von Mises Stress 41

4.4 Compliant MEMS Application 41 
4.5 Challenges

CHAPTER 5 - CONCLUSION

5.1 Discussion of Results and Analysis

44

5.2 Perspective for Future Research

44

APPENDIX

46

REFERENCES

63

ACKNOWLEDGEMENTS 


\section{LIST OF FIGURES}

Figure 1: Flowchart For Compliant Mechanism Design Procedure 8

$\begin{array}{ll}\text { Figure 2: Micro-gripper Sample } & 13\end{array}$

$\begin{array}{ll}\text { Figure 3: Micro-tongs Sample } & 13\end{array}$

$\begin{array}{lr}\text { Figure 4: Micro-tweezers Sample } & 13\end{array}$

$\begin{array}{ll}\text { Figure 5: Micro-vbeam Sample } & 13\end{array}$

$\begin{array}{ll}\text { Figure 6: Orientation-dependent Etch } & 15\end{array}$

Figure 7: Micro-compliant gripper and clamp deflection illustration 22

Figure 8: Illustration of Pseudo-Rigid Body Analysis 28

Figure 9: Parameterization of Design Domain 30

Figure 10: Fabricated compliant micro-gripper and micro-clamp 34

Figure 11: Mechanical and Geometric Advantage Illustration 36

Figure 12: Wet Chemistry Destroying Polysilicon Layer 42

Figure 13: L-EDIT Illustration of Micro-compliant gripper 50

Figure 14: L-EDIT Illustration of Micro-compliant clamp 50

Figure 15: Design domain of compliant gripper 51

Figure 16: FEM Deflection Mesh of compliant gripper 51

Figure 17: Optimize 1/2 micro-gripper structural interpretation 51

Figure 18: Micro-gripper no.2 and no. 4 schematic 52

Figure 19: Micro-gripper no. 1 and no. 3 schematic 52

Figure 20: Micro-gripper fabrication schematic 53

Figure 21: Compliant micro-gripper Stress Distribution 54

Figure 22: Compliant micro-gripper deflection layout 54 
Figure 23: Design domain of compliant micro-clamp

Figure 24: FEM Deflection Mesh of compliant micro-clamp

Figure 25: Optimize micro-clamp structural interpretation

Figure 26: Micro-clamp no. 1 and no. 2 schematic

Figure 27: Micro-clamp no. 3 and no. 4 schematic

Figure 28: Micro-clamp fabrication schematic

Figure 29: Compliant micro-clamp Stress Distribution

58

Figure 30: Compliant micro-clamp deflection layout

58

Figure 31: Test Structure For Device Characterization 


\section{LIST OF TABLES}

Table 1: Comparison Between Bulk and Surface micromachining 16

Table 2: Petersen's Material Comparison 20

Table 3: Dimensions of Micro-gripper Designs 40

Table 4: Dimensions of Micro-clamp Designs 52

Table 5: Yield Profile of Compliant Micro-gripper and Micro-clamp Fabrication 56 


\section{ABSTRACT}

The consideration of compliant mechanisms as Microelectromechanical Systems (MEMS) is the focus of this research endeavor. MEMS are micron to millimeter devices that combine electrical, mechanical, and information processing capabilities on the same device. These MEMS need some mechanical motion or parts that move relative to each other. This relative motion, using multiple parts, is not desired because of the assembly requirement and the friction introduced. Compliant devices limits or eliminates friction and the need for multi-component assembly. Compliant devices improve designs by creating single piece mechanisms. The purpose of this research is to validate surface micromachining as a viable fabrication process for compliant MEMS designs. Specifically, this research has sought to fabricate a microcompliant gripper and a micro-compliant clamp to illustrate the process. While other researchers have created compliant MEMS, most have used comb-drive actuation methods and bulk micromachining processes. This research focuses on fullycompliant devices that use device flexibility for motion and actuation. Validation of these compliant MEMS is achieved by structural optimization of device design and functional performance testing. This research contributes to the ongoing research in MEMS by evaluating the potential of using surface micromachining as a process for fabricating compliant micro-mechanisms. 


\section{CHAPTER 1. INTRODUCTION}

"We believe that the next step in the silicon revolution will be different and more important than simply packing more transistors onto the silicon. We believe that the hallmark of the next thirty years of the silicon revolution will be in the incorporation of new types of functionality onto the chip; structure that will enable the chip to not only think, but to sense, act and communicate as well. This revolution will be enabled by MEMS"

\section{-Sandia National Laboratories}

\subsection{Definition of MEMS}

Using the proven technologies of the Integrated Circuit (IC) industry, research has endeavored to make more dynamic use of the silicon material. With both amazing mechanical and electrical properties, silicon has paved the way for exploration into the fabrication and manufacture of a new family of devices, coined Micro-electro Mechanical Systems (MEMS). MEMS are the product of two modern technologies; microelectronics and micromachining. MEMS technology has taken advantage of both the computational ability of microelectronics and the control capability of microsensors, microactuators, and other micro-devices. The driving forces behind these micro-systems are size, cost, and intelligence of the device.

Though small in size, MEMS are not to be judged by size only, but by functionality and implementation. MEMS technologies are about new ways of creating complex miniaturized electromechanical systems and embedding the electronics during fabrication. A typical MEMS device can be defined as: (1) a 
device consisting of micromechanisms and/or microelectronics, (2) a device that can be batch fabricated, and (3) a device that does not require a great deal of assembly to utilize its functionality (http://www.mems-exchange.org/MEMS/whatis.htm).

\subsection{Problems with MEMS}

When developing MEMS, there are five main points of concern: (1) understanding and controlling the material properties of microstructure polycrystalline silicon films, (2) release of the microstructure, (3) material etch rates, (4) constraints defined by the combination of micromachining and integrated circuit fabrication technology, and (5) practices when packaging the completed devices (Shibaike, 1995; Rai-Choudhury, 1997). MEMS technologies represent a new field that is positioned to revolutionize many products and move forward the development of better micro-component subsystems.

\subsection{Compliant Devices}

When deploying a mechanical device, I hope to achieve motion, good strength, and low cyclic failure. This entails the transmission or use of forces that cause some form of deformation (Snelling \& Erdman, 1974). At the macro-scale, distribution of forces is often accomplished by employing linkages, joints, and other components. In some cases this might require several linkages and configurations to achieve a desired output and functionality. Research has shown that a new family of devices called, "compliant mechanisms" (Midha, 1988) are able to produce desired functionality with just a few components. Thus, my research focuses primarily on creating a device that doesn't require a great deal 
of assembly or joints to utilize its functionality as outlined by the definition of MEMS. The development of "compliant mechanisms" will reduce the number of required parts to a single piece in most cases. In this research, I have developed compliant mechanisms as MEMS using surface micromachining to fabricate these devices.

Compliant mechanisms acquire motion and force transmission by elastic deformation. Compliant designs reduce frictional losses, noise, and the need for lubrication. A reduction in these variables is very important for successful MEMS deployment.

The success of a compliant mechanism is determined by the device configuration. It is most important to develop a suitable topology, shape, and size. Two approaches proposed by G.K. Ananthasuresh (1995) discuss design optimization routines. One such method focuses on a kinematics-based approach when the topology of the compliant mechanism is known at the start and is approximated using a rigid body model. The second approach uses the continuum models and structural optimization algorithms to determine the topology of a fully compliant mechanism satisfying the functional behavior. Several other models have been proposed to provide a more systematic methodology to compute a suitable configuration. These other methods will be briefly discussed in chapter 2 .

In this investigation a micro-compliant gripper and micro-compliant clamp design are constructed. A study of the optimization design process, material selection, design factors, and fabrication processes for both designs are 
investigated. The goal is to build a toolkit to optimize the best design independent of the fabrication process for compliant MEMS. This should facilitate a functional and analytical procedure that will make a seamless conversion process from non-compliant micro-mechanisms to compliant micro-mechanisms.

The optimal compliant mechanism is a compromise between good stiffness and flexibility. If too flexible, it will not transmit a suitable force; if too stiff, it will not deform easily.

\subsection{Research Outcomes}

During this investigation both the micro-compliant gripper and microcompliant clamp were successfully fabricated. However, applying the external force to actuate the devices had a tendency to break the mechanisms. Based on the calculated values for the mechanical advantage (MA) and the geometric advantage (GA), it appeared that around $30 \%$ of the work input was lost at the output. This suggests that $30 \%$ of the system energy is stored in the devices during actuation. Consequently, this stored energy eventually led to the device breakage. Furthermore, more work needs to be done to improve the efficiency. This could be accomplished by improving the complaint mechanism synthesis algorithms as well using polymers as the structure layer for MEMS devices.

The advent of new and more diverse smart materials, compliant mechanisms will become the components of choice. This research will attempt to support the claim that single-component, two-dimensional, fully compliant micromechanisms can be successfully fabricated using surface micromachining. The same design parameters tested by Ananthasuresh (1995) for a bulk 
micromachined fabricated gripper device will be considered in efforts to illustrate surface micromachining as a viable process for compliant MEMS development.

\subsection{Chapter Organization}

This thesis is composed of five chapters. Chapter one provides an introduction to micro-electro mechanical systems (MEMS) technologies along with a brief focus and goal of this research. Chapter two discusses related literature on MEMS devices, fabrication processes, general compliant mechanisms, compliant MEMS, future of compliant and non-compliant MEMS devices, and the comparison of surface and bulk micromachining. Chapter three defines the methodology and procedures, geometrical designs, mask development processes, material properties, problem formulation, and design requirements. Chapter four details the results and analysis of the research conducted. Chapter five contains the conclusion and future work proposals. 


\section{CHAPTER 2. LITERATURE REVIEW}

\subsection{MEMS: Foundation and Fundamentals}

Creation of small high performance machines has always been a dream of scientists and engineers. MEMS technology has transformed this dream into a reality. Born from the integrated circuit industry, MEMS are destined to improve the capability of components ranging from military instrumentation to commercial consumer products. Utilizing the well-tested silicon processing techniques, scientists and engineers can now create very small movable parts and systems. The electronics are fabricated using the standard Integrated Circuit (IC) processes. The micro-mechanical elements are created using micromachining processes, namely, bulk or surface micromachining. Three main assets of MEMS are their size, easy batch fabrication, and seamless integration with microelectronics. These assets of MEMS have produced fully self-contained systems.

MEMS is relatively new technology that exploits the microelectronics infrastructure to create micro-size machines and systems. At the Sandia National Laboratories in New Mexico, scientists have developed technologies that enabled the creation of the mechanical systems on chips and the integration of these systems with on-chip control and communication electronics. Only twenty years ago, most micro-mechanisms were used in micro-sensor related applications. Fan, Tai, and Muller (1987) reported the fabrication of the first movable micro-mechanical structures with joints. This allowed the possibility of rotary and translatory motion amongst different links. Following this early 
achievement, researchers have fabricated a vast array of micromechanical elements to demonstrate the capabilities of their processes and technologies. Some examples of these devices include: microacutators, micromotors, optical devices, communication and information apparatus, scanning probe microscopes, medical equipment, fluidic and chemical analysis systems, and biotechnology devices (Fujita, 1991).

In the development of MEMS technology, it is important to understand that the miniaturization of macro machines is not the most effective way to create MEMS applications and explore their usability. The same physical laws govern both the macro and the micro domain (Fujita \& Gabriel, 1991). Miniaturizing a device can enhance its properties as well as introduce new problems to solve.

When seeking to deploy MEMS, issues of packagability, testability, and reliability are main areas of concern. Researchers have observed five primary sources of MEMS failures: 1) failure by stiction and wear, 2) environmental induced failures, 3) cyclic mechanical fatigue, 4) dampening effect, and 5) delamination. (Ramesham, Ghaffarian, \& Kim, 1999)

MEMS technology will continue to produce products with advantages in performance, reliability, and cost. This emerging technology will lead to several successes and new innovations.

\subsection{Compliant Mechanism Design: Foundation and Overview}

The ability to create motion without rigid links and joints has been a major area of exploration and research. Scientists and engineers have always attempted to exploit flexible mechanisms; however, it was limited to spring-like 
components. Using flexibility to generate motion took root in the 1960's with research endeavors by Shoup (1964), Paros and Weisband (1965), and Tuttle (1967). Though their work provided new venues of insight, there was a need for a methodical approach to design these devices. This gave rise to a class of devices called compliant mechanisms, coined by Midha et al. (1983; 1986; 1993). Compliant mechanisms take advantage of non-rigid bodies to create motion. Compliant mechanisms are flexible structures that generate a wide variety of mechanical motions through elastic deformation. The use of fully compliant mechanisms eliminates rigid links and rigid joints, which in turn cancel or limits friction, backlash, and need to assemble components (Sevak \& McLarnan, 1974; Her, 1986). Compliant mechanisms may consist of both rigid and flexible elements. Its total response to inputs may be influenced by both rigid-body and elastic displacement. Her and Midha (1987) developed a degreeof-compliance concept to describe this mobility of compliant mechanism. Thus, it became important to develop the component for a specific task. The function of a compliant mechanism is determined by its topology, shape, and size that determine its ability to deform or deflect in a desired manner.

Desirable features such as single piece construction and easy fabrication make compliant mechanisms usability very feasible and desirous. The flexure in a structure can be further exploited to generate an even greater array of motions (Norton, 1991). By choosing the appropriate configuration, a designer can generate several outputs for a given applied loading (Murphy, Howell, \& Midha, 1994). Sevak and McLarnan (1971) added that compliant mechanisms take 
advantage of stored elastic energy to eliminate the need for multiple components. By reducing the number of components and required interconnections, the reliability of a design is often improved and offers component savings over conventional mechanism designs. Compliant mechanisms can often be joined in series or parallel to build a system of components (Murphy, Howell, \& Midha, 1994). Murphy, Howell, and Midha (1994) developed a process/procedure flowchart (see Figure 1) that can be applied to construct compliant devices for deployment.

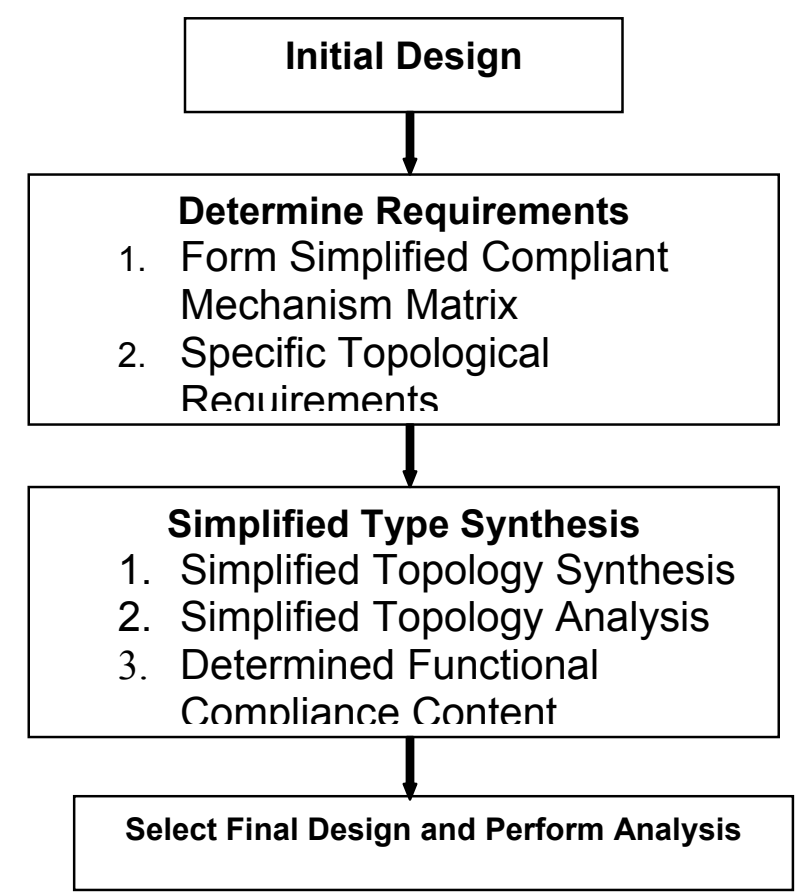

Figure 1: Flowchart For Compliant Mechanism Design Procedure

There are basically two design criteria to satisfy when designing compliant mechanisms: (1) stiffness to maintain structural rigidity and (2) flexibility to meet kinematics requirements (Ananthasuresh, Kota, \& Gianchandani, 1994). These are antithetical objectives. When designing a flexible component, the support for external loads may not be acceptable. Whereas designing a stiff component may 
not yield a desired deflection. A commonality between stiffness and flexibility must be effectively determined in compliant mechanism designs. To reach this critical design point, a suitable topology, shape, and size is necessary. In an effort to develop a synthesis procedure to construct these compliant components several mathematical models have been developed.

\subsection{Topological Synthesis and Development of Compliant Mechanisms}

In general, the deflection of the flexible members of compliant mechanisms is large, and nonlinear analysis methods must be employed to accurately predict their behavior. In many cases, multiple boundary conditions must be considered. A variety of different mathematical models are used to develop an optimization routine to create the best possible design. However, the difficulty with posing mathematical optimization problem is that the formulation may be under-constrained; meaning the solution to the problem does not yield a physically realizable nor correct answer. The formulation may also be overconstrained, meaning no solution will satisfy all the design constraints (Save \& Prager, 1985). In support of this point, Hetrick, Kikuchi, Kota (1999) stated that objective functions, which attempt to simultaneously maximize stiffness as well as flexibility of compliant mechanisms, could be formulated such that they are not well bounded. They analyzed a cantilever beam model to develop a new objective function of maximizing the energy throughput of such devices and demonstrated a well-bounded solution (Save \& Prager, 1985). When deploying compliant devices, the designer must consider that the best possible 
configuration will take in account selection of material, fabrication processes, shapes, and layouts (Burgess et al., 1997).

One such approach used by Salomon \& Midha (1992) and Howell \& Midha (1999) relies on the manipulation of the constraints of a rigid body element. It primarily uses knowledge of kinematics and the Euler-Bernoulli beam theory. They created the rigid mechanisms shapes and replaced the rigid joints with a flexible segment. This is the pseudo-rigid-body mechanism method. This method continues an iterative procedure until the requirements are satisfied based on a set of pre-determined optimization constraints. Such a method is well suited for mechanisms consisting of both flexible and rigid components, i.e., semi-compliant mechanisms. Paros and Weisbord (1965) introduced fundamentals for designing flexure hinged mechanisms, Boronkay and Mei (1970) used numerical methods such as nonlinear large-deflection finite element methods, Burns and Crossley (1973) investigated synthesis techniques for mechanisms with flexible coupler links, Solomon (1989) and Norton (1991) studied the concept of pseudo-rigid body model for flexible members, Mettlach and Midha (1996) proposed using the Burmester Theory in the design of compliant mechanisms. These methods will not completely satisfy the design goals in this research proposal. This research investigation focuses primarily on fully compliant mechanisms, however; it is important to introduce these models to provide a comprehensive approach to compliant mechanism design.

Ananthasuresh, Kota, and Kikuchi (1994) and Frecker et al. (1997) introduced a four-step synthesis procedure for designing fully compliant 
mechanisms. These steps include (1) minimizing compliance for a given weight, (2) maximizing compliance for a given weight, (3) minimizing deflection at the point of interest for a given weight, and (4) minimizing deflection at the point of interest in conjunction with bounds of compliance. Based on these steps, they developed two models, a spring model and a multi-criteria/multi-purpose model. These models allowed for an evaluation and comparison of pre-determined performance criteria that the compliant mechanism must meet. This thesis adopts the multi-criteria formulation and the homogenization method (Bendsoe \& Kikuchi, 1988) to compute the desired topology, shape, and size. The multicriteria formulation includes both stiffness and flexibility considerations. With these two properties being very important in compliant mechanism designs, the formulation proposed by Ananthasuresh (1994) is considered in the synthesis of both the compliant micro-gripper and the compliant micro-clamp.

\subsection{MEMS as Compliant Mechanisms}

Compliant mechanisms can be easily miniaturized using existing microfabrication processes and thus are best adaptable for MEMS. Because compliant designs eliminate the need for assembly and in most cases reduce or eliminate friction, these are very suitable options.

In the micro regime, it is important for a design to meet the typical mechanical criterion as well as the dominating issues at the micro level, namely, dust particle contamination and device buckling. When scaling down the size of the mechanisms, mechanical laws also affect its behavior (Fujita \& Gabriel, 1991). Thus, the use of such flexible elements is very much desirable. Also, the 
ability to generate a desired motion via deformation is becoming very important with the growth of smart material deployment and usage. Though historically flexible devices are best suited for low speed and short-range applications (Davidson, 1970), MEMS configurations can successfully support higher speeds and increasing ranges of motion. This improvement is the result of the scaling down of the components. For example, torque transmission and heat transfer improves as the devices are miniaturized. This improves the characteristics of the fatigue of the components. With such great benefits from this mechanical standpoint, compliant mechanisms are great consideration for MEMS adaptability.

\subsubsection{Related MEMS Designs}

Adopting the mature fabrication processes of the integrated circuit industry, researchers have explored various developments of semi-compliant micro-clamp and micro-gripper type devices. Kim, et al. (1990) at the University of California developed and tested a polysilicon, electrostatic, comb-drive microgripper. They were able to create a cantilever comb-drive arm with a bidirectional actuation scheme and over-range protectors. These over-range protectors restricted the arms from moving too far apart. This device was tested by applying a voltage across the comb-drive and by measuring the gripper tip change in separation. Kim, Pisano, and Muller (1990) expanded on the first design of the microgripper to fabricate a silicon-processed overhanging microgripper, see Figure 2. This design change was to overcome the problem in positioning objects for actual gripping. The small gap between the suspended 
gripper and the substrate limited the mobility of the objects. This overhanging gripper is free of the substrate. It is mounted on a maneuverable silicon die. A cantilever support protrudes from the die to serve as the base for the gripper structure. The support cantilever, heavily doped with boron, is defined geometrically by etch-stopping in ethylenediamine pyrocatechol (EDP). The anisotropic etching EDP must be controlled to prevent the rounded edges from meeting underneath the $p+$ region, see Appendix. This would lead to rapid edge propagation. Mehrany, Gabriel, and Trimmer (1988) fabricated polysilicon tongs that were actuated by external means (see Figure 3). More related works by Chen et al. (1989) illustrated the actuation of tungsten micro-tweezers (see Figure 4). Saggere (1996) successfully fabricated a V-Beam micro-suspension system utilizing surface micromachining (see Figure 5). Jensen, Howell, Salmon (1998) successfully fabricated and tested a two-link, in-plane, bistable compliant MEMS device.

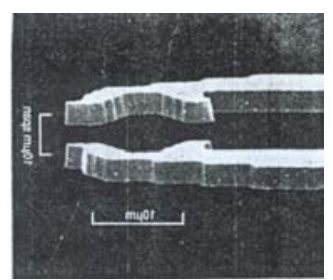

Figure 2: Micro-gripper (Kim, et al 1990)

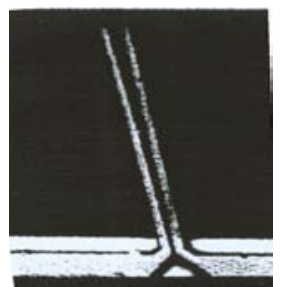

Figure 4: Micro-tweezers (Chen, 1989)

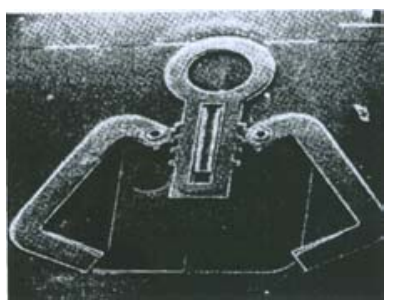

Figure 3: Micro-tongs (Mehrany, et al 1989)

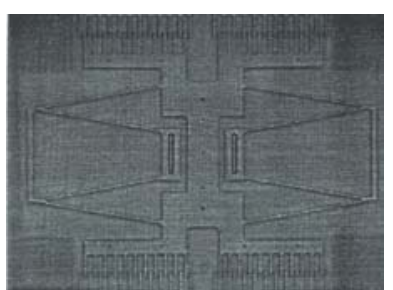

Figure 5: Micro-Vbeam (Saggere, 1997)

The fabrication and operation for most of these devices utilize the electrostatic comb-drive actuation method. This method was selected because of 
its stable drive over relative long distances. This differs in relation to other variable gap capacitors. Most variable gap capacitors are better suited for short distances and can become unstable for longer distances.

The aforementioned devices have both compliant as well as rigid components. In essence these devices successfully qualify as semi-compliant mechanisms. Most of them were fabricated using the bulk micromachining process. This thesis proposes surface micromachining as viable method for a fully compliant mechanism. A fully compliant mechanism will eliminate all rigid segments and the need for a comb-drive actuation method.

\subsection{Microfabrication Process: Bulk and Surface Micromachining}

Micromachining refers to the fabrication of small structures by a series of etching and/or deposition processes on a substrate or thin film. The Honeywell Corporation successfully fabricated the first silicon pressure sensor using isotropic etching. This process used both wet and dry etching as well as silicon oxidation. These crystal orientation-dependent etchants lead to very precise structure formation.

Bulk micromachining was the process of choice during the early days of microfabrication, i.e. 1960's. However, the early nineties ushered in a new era and a new process, surface micromachining.

Bulk and Surface Micromachining two technologies have continued to develop in simultaneously synchronization. French and Sarro (1998) investigated these two technologies and compared their (1) properties, (2) processing, and (3) implementation. 
Bulk micromachining etches through the backside of the wafer to fabricate the structures. Note that this structure uses wet anisotropic etchants, see Figure 6. These etchants are dependant upon the crystal orientation, commonly used in bulk micromachining. In some cases, it might be necessary to stop the etching process to maintain a particular dimension for a component. This can sometimes be empirically determined based on the etch rate and thickness of the wafers. Based on the calculated etch time, wafers are removed from the etchant material. However, this method can sometimes produce inaccuracies. There are new methods used today such as EDP (etch stop) or maybe an electro-chemical etch stop.

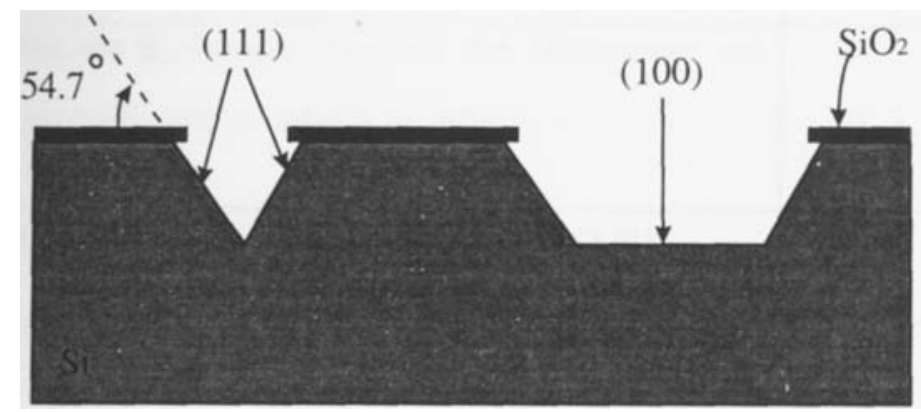

Figure 6: Orientation Dependent Etch

Surface micromachining deposits and/or removes thin films from the silicon substrate as opposed to forming the structure in the silicon substrate as in bulk micromachining. Surface micromachining usually grows a sacrificial layer on the surface of the substrate. This layer is patterned, followed by a similar process for the mechanical layer, usually polysilicon deposition and patterning. The final step is the removal of all sacrificial layers and release of the component. This process introduces a challenge to find an etchant that will remove the sacrificial layer without damaging the mechanical components. 
Now after this brief discussion of the two most used microfabrication processes, surface and bulk micromachining, it is important evaluate some of the characteristics that can help decide when to use the appropriate process. I investigated a few qualitative concerns between these two processes, namely dimensional limitations; yield ratios, compatibility with electronics as well as manufacturability.

As illustrated in Table 1, the dimensions of the structures produced by these two rival processes appear to show some considerable differences in both lateral and vertical dimensions (French \& Sarro, 1998). It appears that the bulk micromachining process lends itself well to creating very thick structures that are smaller in lateral length. The surface micromachining process produce structures that appear to be quite opposite, smaller in thickness and can be designed to have very large lateral lengths.

Table 1: Comparison between bulk and surface micromachining (courtesy French and Sarro)

\begin{tabular}{|l|l|l|}
\hline Property & Bulk Micromachining & Surface Micromachining \\
\hline Processing Complexity & 0 & 0 \\
\hline Lateral Dimensions & $3-5 \mathrm{~mm}$ & $100-500 \mu \mathrm{m}$ \\
\hline Vertical Dimensions & $100-500 \mu \mathrm{m}$ & $0.5-2 \mu \mathrm{m}$ \\
\hline
\end{tabular}

The yield ratio is very important for product development. For a product to be marketable, it is necessary to have a very high yield rate of successful components. Both bulk and surface micromachining present challenges to insure a high yield. For instance, bulk micromachining can result in devices with variations in thickness (Kovacs, Maluf, \& Petersen, 1998). These variations can cause a device to be out of specification due to etch stops. Surface micromachining also introduces these thickness variations during each 
deposition of a new layer (Bustillo, Howe, \& Muller, 1998). For surface micromachining, this can result in variations in mechanical and electrical properties. Furthermore, these deposited layers typically have intrinsic stress. A difference in stress on the top and bottom surface of a structure can cause considerable bending. Similar stress problems are also found in heavily borondoped structures.

When combining both microstructures and the microelectronics on a single chip, the process and the structure design must be optimized. Bulk micromachining usually introduce problems due to etching at intermediate steps in the process. In some cases, etching can render the wafer surface unsuitable for any subsequent steps. However, this can usually be overcome by designing a process that will only etch at the end of the fabrication phase. Surface micromachining typically introduces compatibility problems as a consequence of several depositions, annealing, etching, and masking steps. In some cases, the etchant used to remove sacrificial layers has a negative effect on the metal material. In this investigation, surface micromachining will be used for the fabrication process; however, fabricating compliant designs can greatly reduces the need for numerous deposition and removal steps. This behavior also supports surface micromachining as a viable process for compliant MEMS fabrication (Burgess, Moore, Newland, \& Klaubert, 1997).

\subsection{The Future of MEMS Technologies and Applications}

Miniaturization of macroscopic machines is not the best strategy for the development and deployment of MEMS. It is obviously no comparison to the 
strength and power of conventional mechanisms and machines. For the successful development of MEMS in the future, it is important to avoid mechanical coupling with the outside world while trying hard to improve technology and enhance the mechanical power of the devices (Boa \& Wang, 1996). The focus for MEMS should be the creation of new technologies and applications, not simply reconfigurations of traditional mechanisms.

In 1987, the first movable micromechanical parts were created by surface micromachining technology (Fan, Tai, \& Muller, 1987). This breakthrough led the way in development of new processes and techniques such as: sacrificial LIGA, lithography on 3D surfaces, and low temperature bonds. These new processes have led to the creations of comb drivers, wobble motors, micro-tweezers, microgripper, micro-clamps, and several other devices.

To continue moving forward in MEMS, (1) new micromachining and fabrication technologies must be developed; (2) appropriate uses and deployment schemes must be created (Spearing, 1999). Following these two objectives will create a very efficient system to bring these new technologies to market and increase usability.

Though MEMS provide several advantages such as: low cost, batch production, and high precision, there are always limitations. Limitations of MEMS are low strength, low power output, and environmental contamination (Ramesham, Ghaffarian, \& Kim, 1999). MEMS should be packaged as independent subsystems with coupling using electrical, optical, magnetic, or some other technique to communicate with the outside world (Fujita, 1998). 
As research grows, MEMS will continue to be found in inertial sensing devices, optical signal processors and display, magnetic signal output, micropositon controls, chemical and biochemical analysis and reactions, scanning probe microscopes, smart homes, and several other applications.

The success of MEMS will be tied to three important factors (1) offering of easier access to information, (2) making human life more compatible with the outside world, and (3) improving social welfare. Developments to accomplish these goals can be broken down into five general areas: machine intelligence, downsizing and parallelism, biomimetics, informatics, and environment monitoring (Fujita, 1998). These developments will allow easier collection, analysis, and dissemination of information on demand. Fuzzy logic technology can adapt to the micro regime with the introduction of flexible and sensitive MEMS. Friction and tribology, in the micro regime, must be well understood. MEMS teaching and instruction will need to encompass knowledge from a wide array of scientific and engineering domains including inorganic and organic material science, process engineering, computer science, embedded systems, and a variety of other fields. A standardized methodology for testing, evaluating quality and reliability, and packaging must be established to provide more credibility to the development and deployment of such devices.

As smart materials become more robust and versatile, compliant MEMS will become a vital part of micro-mechanisms design. This thesis discusses surface micromachining as a possible fabrication process for compliant mechanisms. Compliant mechanisms will allow the user to design a device using 
a smart material. Such designs will take advantage of both properties of device compliance as well as properties of smart material (Frecker, 1997).

MEMS will have a great effect on the future of product development. It is very important to continue research on new techniques and applications for MEMS. For continued success, collaboration amongst a broad spectrum of scientific and engineering disciplines must be established and well promoted. 


\section{CHAPTER 3. METHODOLOGY AND PROCEDURES}

\subsection{Geometrical Design}

When designing micro mechanisms, it is important to understand the material and fabrication processes, as well as geometrical designs. There are three important design factors to consider (1) device configuration, (2) available material, and (3) available fabrication processes (Shibiake, 1995).

Since MEMS are basically developed on silicon substrates, silicon mechanical properties and characteristics must be understood. The Petersen's table below provides a comparative list of mechanical characteristics of single crystal silicon with other materials.

Table 2: Petersen's Material Comparison

\begin{tabular}{|c|c|c|c|c|c|}
\hline Material & $\begin{array}{l}\text { Yield Strength } \\
\text { (10E12dyn/cm) }\end{array}$ & $\begin{array}{l}\text { Youngs } \\
\text { modulus } \\
(10 \mathrm{E} 12 \mathrm{dyn} / \mathrm{cm}\end{array}$ & $\begin{array}{l}\text { Density }(\mathrm{g} / \mathrm{cm} \\
\text { ) }\end{array}$ & $\begin{array}{l}\text { Thermal } \\
\text { conductivity } \\
\text { (W.cmC) }\end{array}$ & $\begin{array}{l}\text { Thermal } \\
\text { Expansion } \\
(10 \mathrm{E}-6 / \mathrm{C})\end{array}$ \\
\hline Diamond & 53 & 10.35 & 3.5 & 20 & 1.0 \\
\hline $\mathrm{SiC}$ & 21 & 7.0 & 3.2 & 3.5 & 3.3 \\
\hline $\mathrm{Al} 2 \mathrm{O} 3$ & 15.4 & 5.3 & 4.0 & .5 & 5.4 \\
\hline Si3N4 & 14 & 3.85 & 3.1 & .19 & .8 \\
\hline Iron & 12.6 & 1.96 & 7.8 & .803 & 12 \\
\hline SiO2(fibres) & 8.4 & .73 & 2.5 & .014 & .55 \\
\hline $\mathrm{Si}$ & 7.0 & 1.9 & 2.3 & 1.57 & 2.33 \\
\hline Steel(max.) & 4.2 & 2.1 & 7.9 & .97 & 12 \\
\hline W & 4.0 & 4.1 & 19.3 & 1.78 & 4.5 \\
\hline Stainless Steel & 2.1 & 2.0 & 7.9 & .329 & 17.3 \\
\hline Mo & 2.1 & 3.43 & 10.3 & 1.38 & 5.0 \\
\hline $\mathrm{Al}$ & .17 & .70 & 2.7 & 2.36 & 25 \\
\hline
\end{tabular}

The research in this thesis is based on the fabrication of compliant MEMS mechanisms using surface micromachining. Compliant mechanisms, by their 
design, generate a desired motion through elastic deformation. The flexure of such mechanisms can be exploited by choosing the appropriate topology, shape, and size for the structure. This research attempts to fabricate a compliant microgripper and micro-clamp (Cho \& Pisano, 1992), using surface micromachining based on dimensions from the MCNC and the homogenization method (Bendsoe \& Kikuchi, 1988) proposed by Ananthasuresh (1995). Each of the devices is evaluated using specified design criteria. Design criterions and performance measures considered in this research are mechanical advantage (the ratio of force output to force applied), compliance of the mechanisms (work done by the external forces), acceptable stress and strain values, buckling and loading characteristics, and manufacturability. Compliant MEMS can satisfy many problems of conventional microfabricated components such as: (1) restrict the entire machine system to just one or two layers in a plane as opposed to the multi-layer required designs, (2) alleviate friction, (3) alleviate assembly, (4) and accommodate unconventional actuation techniques including thermal and piezoelectric processes (Frecker, Kota, \& Kikuchi, 1998). This research will evaluate these criteria in an effort to substantiate the fabrication of compliant MEMS using surface micromaching.

A micro-gripper was chosen to illustrate the fabrication process. The operation of the compliant micro-gripper is such that the gripper arms close together when an external force is applied at the mid-connector as shown in Figure 7. This figure shows the direction of the applied force and the corresponding movement of the gripper. A micro-clamp has also been 
considered. The operation of the clamp is such that the arms of the clamp separate when an external load is applied at the two notches in the clamp arms as illustrated in Figure 7. The figure shows two forces applied in opposite and parallel direction and results in the opening of the clamp. Both of these components have the inherent ability to return to the original configuration after removing the applied loading.

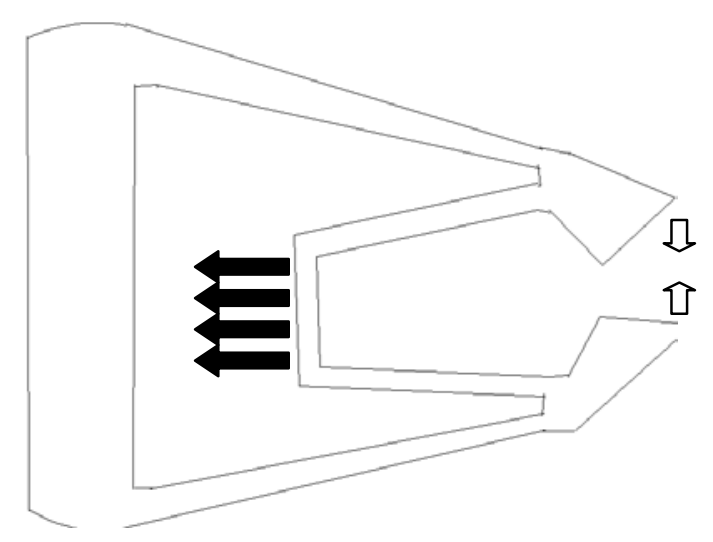

Micro-Compliant Gripper

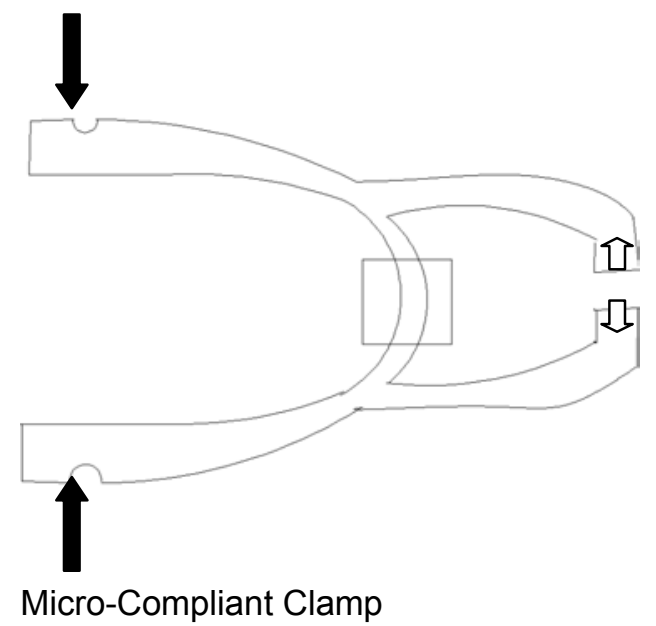

Figure 7: The shaded arrows represent the direction of the applied force. The unshaded arrows represent the direction of deflection and deformation.

The functional output is the goal in compliant designs, meaning for an applied force on a given structure a desired deflection or output is realized, Midha (1992). To evaluate this functional behavior, simple tests are conducted to evaluate for each design. These test and analysis are detailed in the following chapter four.

\subsection{Problem Formulation}

The difficulty to assemble components at the micro-level makes compliant MEMS devices a viable solution for MEMS designers. Ananthasuresh, Kota, \& Kikuchi (1994) have successfully demonstrated the performance of a microgripper fabricated using the bulk micro-machining process. They conducted 
simple performance analysis to assure that the component actually works. They also performed simulation processes to calculate the mechanical advantage for the device. In this thesis, an attempt has been made to fabricate both a microgripper and micro-clamp as a freestanding device using the surface micromachining technique. Also, recalling from chapter two was noted several examples of micro-gripper and micro-clamp type devices, however; those devices were fabricated using bulk-micromachining. They also took advantage of comb-drive actuation methods instead of device flexibility. This research is an effort to substantiate the claim that compliant mechanisms can be successfully fabricated using both current processes, namely bulk and surface micromachining. To justify this proposal, investigations were conducted to determine suitable performance by testing whether or not the micro-device exhibits simple functionality. Also, this research will quantify the mechanical advantage, develop a finite element analysis model, and discuss the topology optimization model.

\subsection{Determination of Design Requirements}

The analysis of the mechanism and the specification of design requirements are critical steps in the design process. As with many mechanism designs, this process is dependent upon the use and application of the device. In this research endeavor, the compliance of the design was considered, meaning a device that is both flexible and stiff enough to satisfy the design criterions, as well as the small shape and size, meaning that this device will be measured in units of microns and created using microfabrication processes. When considering the 
compliance, a designer must realize an acceptable topology and predict the functional behavior. Furthermore, surface micromachining differs significantly in its allowable dimensions for device designs in comparison to bulk micromachining. These differences must be considered when developing the design concept. Viewing the designs presented in this research and the designs by Ananthersuresh (1995), illustrates a difference in the dimensional values and physical design of the devices. This study began with a known design domain for the optimization and used micro-fabrication processes to construct the microcompliant gripper and micro-compliant clamp.

\subsection{Surface Micromachining Procedures in MEMS Fabrication}

In the following three sections briefly discuss some of the important factors and design procedures in MEMS fabrication. These processes were used in the creation of both the design mask as well as the actual released polysilicon structure on the silicon substrate.

\subsubsection{Mask Development and Design}

The first stage in the generation of the micro device is the creation of a photomask. Exposing the desired pattern in a resist layer spun on top of a chromium layer creates a photomask. The pattern is developed in the resist layer and the chrome layer is etched through the pattern in the resist. The resist is removed. This releases the pattern. This process is covered in nine principal fabrication steps: 1) substrate preparation, 2) pattern writing, 3) pattern processing, 4) metrology, 5) inspection for pattern integrity, 6) cleansing, 7) repair, 8) pellicle attachment, and 9) final defect inspection. Throughout the 
process, consideration must be taken to ensure that mask specifications are met such as pattern position accuracy, feature size control, and defect density.

Using the L-Edit software application, both the micro-clamp and the microgripper were created from a series of small rectangles and tapered edges to produce a design to feed into the mask-processing machine. From Figure $13 \&$ 14 (Appendix), it is shown that the distance between the grid points are 10 microns apart. The process steps were to create only the top half of the component and reflect the component about the x-axis to create a symmetrical design. Two masks for each design were created. One design was used to construct the mounting post. The other design is the actual gripper and clamp mechanism with changes only to the dimensions.

\subsubsection{Fabrication Processes and Technique}

After completing the cad modeling and mask design, it is now time to proceed to the actual fabrication process. There are nine general steps to follow when developing these microsystems using surface micromachining: (1) initial wafer cleaning, (2) sacrificial layer deposition, (3) photoresist coating, (4) photolithography exposure, (5) polysilicon deposition, (6) polysilicon etch, (7) photoresist stripping, (8) metallization, and (9) finish product (MCNC, 1992). Each of these steps has a specific purpose. The initial wafer cleaning is performed prior to any film deposition. In this procedure, a number of acids were used to clean the wafer. This will ensure that all organic and metallic contaminants are eradicated from the wafer substrate. After the cleaning process, a sacrificial layer is deposited on the wafer. After deposition, it is 
important to determine the layer thickness and uniformity on the substrate, measuring the layer using a nanospec. Now that the wafer (substrate) has passed the inspection test, a photoresist coat is applied to the wafer. This photoresist is a very light sensitive polymer. After depositing the photoresist on the wafer, it is now time to transfer the pattern on the photomask to the wafer substrate. At this point, the wafer is exposed with ultra-violet light through the photomask to transfer the pattern to the photoresist. Once the photoresist is patterned, the pattern is then transferred to the deposited film on the wafer substrate using wet or dry etching techniques. Now that the etching is completed, the photoresist is stripped leaving the patterns in the films. Following each step in the process eventually leads to a polysilicon deposition. These polysilicon films are used as the mechanical structure layers. It is essential to inspect the wafers to ensure that all alignments are correct which would effect aligning any multilayer MEMS designs. The next step is to check that the pattern is accurate and meets certain critical dimensions. The wafers have to essentially meet the same criteria as the parent photomask. The next step is metallization, if required. The final step is the removal of the sacrificial layer to release the component. This is essentially the construction of MEMS.

Regarding other materials that are deposited, silicon nitride can be used for electrical or chemical isolations, and phosphosilocate glass (PSG) or silicon oxide can be used as a sacrificial layer.

Using these standard fabrication processes, this research has proposed to create a micro-gripper and micro-clamp using surface micromachining 
techniques, section 3.5. Small test probes (piezo-electric transducers) are used to test the functional behavior as described in section 3.2. Optimization has been considered by designing the device in mind of mechanism compliance and available fabrication characteristics and processes. Test results and Finite Element Analysis are included in the following chapters four and five.

\subsubsection{Material Properties}

The material issues in MEMS are divided into four categories, the MEMS (1) material set, (2) microfabrication processes, (3) material characterization, and (4) design (Spearing 1999). The micro scale of MEMS affords the opportunity to use materials not economically feasible in the macro regime (Shibaike, 1995). The materials set for MEMS fabrication has been primarily defined by the three most widely used fabrication processes; surface micromachining, bulk micromachining, and molding. Silicon, polysilicon, and silicon nitride are used for mechanical components, aluminum and copper for the electrical conductors, and silicon dioxide as a sacrificial layer. Having a limited set of materials creates a controlled environment that ensures reproducibility and compatibility with the onchip electronics. Elasticity, high strength, and past successes make these materials very desirable (Spearing, 1999). It also has a relatively high yield and fracture strength. However, low toughness of silicon makes it a little less than ideal for mechanical devices. Materials such as diamond, aluminum oxide, and silicon carbide are being considered as substantial substrate choices; however, techniques to etch these materials are not completely defined. 


\subsection{Design and Fabrication For The Compliant Micro-gripper and Micro- clamp}

In this section, I will discuss the compliant MEMS design and fabrication process. This section outlines the implementation of the homogenization method (Bendsoe \& Kikuchi, 1988) and the pseudo-rigid body model for MEMS design (Howell \& Midha, 1995). It also provides the step-by-step process for the fabrication of the micro-gripper and micro-clamp.

\subsubsection{Design Methodology For The Compliant Micro-gripper and Micro- clamp}

Because one of the most important attributes of a compliant design is the structural configuration, it is essential to develop a design that will produce the desired output deformation. In this investigation, I have attempted an implementation of both the homogenization method (Bendsoe \& Kikuchi, 1988) and the pseudo-rigid body model (Howell \& Midha, 1995). Because I had to consider the design constraints introduced by the surface micromachining process, structural dimensional differences between Ananthesuresh (1995) and this investigation are observed. These design constraints were based on my intuition after thorough investigation of literature on surface micromachining processes and characteristics.

The pseudo-rigid model implementation (Murphy, Howell, \& Midha, 1994) began with a known design and performed general analysis. A general free-body diagram illustrates applied forces and constraints to simulate the device 
behavior. Furthermore, This process is an attempt to illustrate, show, and analyze the

designs implemented by Ananthesuresh (1995).

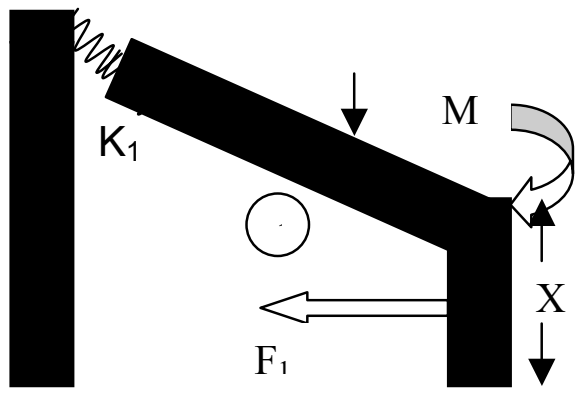

(a) $\mathrm{M}_{1}=\mathrm{F}_{1} \mathrm{X}_{1} ; \mathrm{K}_{1} \Delta \mathrm{s}$

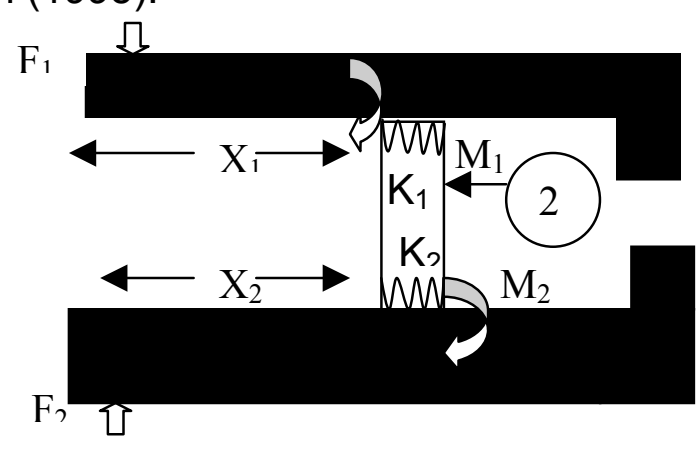

(b) $\mathrm{M}_{1}=\mathrm{F}_{1} \mathrm{X}_{1}$ and $\mathrm{M}_{2}=\mathrm{F}_{2} \mathrm{X}_{2} ; \mathrm{K}_{\mathrm{i}} \Delta \mathrm{s}$

Figure 8: Illustration of Pseudo-Rigid Body Analysis for (a) gripper and (b) clamp design. Beam 1 illustrates a cantilever behavior and Beam 2 illustrates a bending behavior.

Figure 8 illustrates a pseudo-rigid body model constraints and force implementations, (a) the compliant micro-gripper and (b) a compliant microclamp. To model the flexibility of compliant devices, springs are added to the models. This introduces a spring constant, "k". As noted in the figure, the external forces are applied to the devices at the desired location. This will induce a more cantilever-like affect in beam 1; however, beam 2 will undergo more of a beam bending behavior. Adding the springs to the models allows a method to solve for the moments and deflections using the general beam equations for compliant mechanisms. Consider the micro-gripper. The moment about Beam 1 can be approximated using the following equation: $M_{1}=\left(F_{1}-K_{1} \Delta s\right) X_{1}$; where $F_{1}=$ externally applied force, $K_{1}=$ spring constant, $\Delta s=$ change in spring length due to applied force, and $X_{1}=$ location of external applied force. The moments about Beam 2 can be approximated using similar formulations as well. 
In the homogenization method, analysis is used to first determine the function of the device in terms of the input forces, output deformations, and available design domain. The design domain determined the amount of material available for design. In this case, the design domain has a maximum $\mathrm{x}$-direction of $2 \mu$ and y-direction $4 \mu$ and a maximum z direction (thickness) of $1.5 \mu$ to $2.0 \mu$. Figure 15 illustrates the gripper design and figure 23 illustrates the clamp design. This thickness was chosen to prevent buckling which is often common in high aspect ratio MEMS designs. The homogenization method employs a technique that uses cellular microstructures. This method works by solving for a series of parameters $(\alpha, \beta$, and $\theta)$ for each cell, see figure 9 . The values of these parameters determine the characterization of the design space. This means that a hole is created when parameters reach a prescribed upper bound and a porous or solid region is created when the parameters fall within the lower and upper bounds. This is shown in figures 17 and 25 . These figures show the removal of some elements by the optimization routine discussed by Ananthesuresh (1995) and programmed by Sarma (1997).

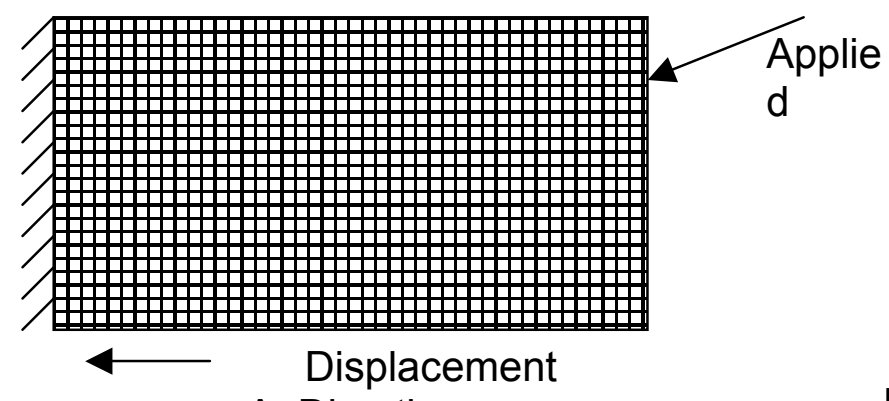

A. Direction

B.

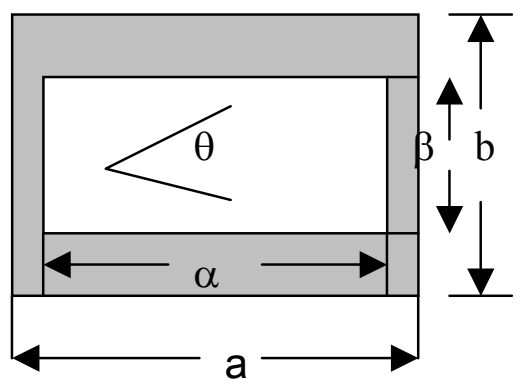

Figure 9: Parameterization of the design domain (A) with the microstructural cells having rectangular holes (B) 


\subsubsection{Fabrication Process For The Compliant Micro-gripper and Micro- clamp}

Process Steps and Fabrication

To fabricate this compliant micro-gripper and micro-clamp, I developed a process to fabricate a $1.5 \mu$ to $2.0 \mu$ thick device mounted on a $.85 \mu$ post attached to a silicon substrate. Initially, I had to analyze the device geometry and configuration.

\section{Processing:}

1. Thermally grow a $.85 \mu$ silicon dioxide $\left(\mathrm{SiO}_{2}\right)$ on the silicon substrate. I placed the 12 wafers in a stream of water vapors for 1.1 hours at $1200^{\circ}$. These wafers are 3 inches in diameter, $<100>$ orientation, resistivity of 1 $10 \Omega-\mathrm{cm}$, and thickness $356-406 \mu \mathrm{m}$. This oxide is governed by the following chemical reaction of silicon immersed in a water vapor stream:

$\mathrm{Si}\left(\right.$ solid) $+\mathrm{H}_{2} \mathrm{O}$ (vapor) $\longrightarrow \mathrm{SiO}_{2}$ (solid) $+2 \mathrm{H}_{2}$ (gas)

The thickness of the oxide is governed by the following equations: $X_{o x}+A X_{o x}=B(t+\tau)$, where $X_{o x}$ is the oxide thickness and $B / A$ is the linear rate coefficient, $(t+\tau)$ is the total effective oxidation time, and $\mathrm{B}$ is the parabolic rate coefficient. Here is a sample calculation for our process:

Desired thickness $=0.85 \mu \mathrm{m}$

$\mathrm{B} / \mathrm{A}\left(\right.$ at $\left.1200^{\circ}\right)=14.6529 \mu \mathrm{m} / \mathrm{hr}$

B $\left(\right.$ at $\left.1200^{\circ}\right)=0.7032 \mu \mathrm{m}^{2} / \mathrm{hr}$ $\tau=0$

Now solve for " $\mathrm{t}$ "; $\mathrm{t}=\left[\frac{(.85)(.85)}{.7032}+\frac{.85}{14.6529}-0\right](60) ; \mathrm{t}=65.0235 \mathrm{~min}$. 
2. Apply HMDS adhesive and AZ5214 photoresist. The photoresist is spincoated on the $\mathrm{SiO}_{2}$ layer.

3. Photolithography process to pattern the oxide. This process was used to pattern the post on the $\mathrm{SiO}_{2}$ layer. Step 2: Exposing the post regions. This post region will serve as the anchor points for the clamp and gripper mechanisms.

4. Etch the silicon dioxide using Buffer Oxide Etchant to remove the exposed oxide. We also strip the photoresist. This results in the silicon dioxide anchoring post on the silicon substrate.

5. PECVD oxide deposition to create a hard-mask for patterning the subsequent polysilicon layer. (optional step)

6. $1.5 \mu$ to $2.0 \mu$ Polysilicon deposition and post annealing process to depress the stress in the polysilicon. The stress in the polysilicon layer was reduced from an average of 200 MPA to approximately 25.7 MPA after the annealing process. The polysilicon layer determines the thickness of the clamp and gripper devices.

7. Spin photoresist on the substrate. Expose and develop the gripper and clamp patterns on the polysilicon layer.

8. Etch the polysilicon using the reactive ion etching process and wet chemistry solution of $\mathrm{HNO}_{3}+\mathrm{HF}+\mathrm{CH}_{3} \mathrm{COOH}+\mathrm{H}_{2} \mathrm{O}(\mathrm{DI})$.

9. Removal of the photoresist.

10. Removal of the sacrificial silicon dioxide to release the gripper and clamp mechanism using BOE (Buffered Oxide Etch). This process is an isotropic 
etch process, meaning that the etch rate is equal in all direction. In this case, the micro-gripper and micro-clamp where undercut in all direction at an approximate etch rate of $400-500 \mathrm{~A} / \mathrm{min}$. For the dimensions of these device, the etch time was approximately $7 \mathrm{hrs}$. to release. This BOE process can be compared to a diffusion process, which is governed by Fick's Law:

$J=-D \frac{d N(x)}{d x} ;$ Fick's Law. This law defines the movement of the diffusing material in terms of flux $(J)$, which is the number of particles moving through a unit area in a give time, $\frac{d N(x)}{d x} . D$ is defined as the diffusion coefficient. The BOE process resembles diffusion into the oxide layer. Note that etch characteristic are different for thermally grown oxide layers and deposited oxide layers.

Thus, this process is governed by the following chemical reaction:

$\mathrm{SiO}_{2}+6 \mathrm{HF} \rightarrow \mathrm{H}_{2}+\mathrm{SiF}_{6}+2 \mathrm{H}_{2} \mathrm{O}$

This reaction is the process that the wafer is undergoing when submersed in the BOE solution. 


\section{CHAPTER 4. RESULTS AND ANALYSIS}

\subsection{Description of Fabricated Micro-Gripper and Micro-Clamp}

A compliant micro-gripper and micro-clamp were fabricated using the process steps presented in chapter three. Each device was suspended on a post attached to the silicon substrate, see Figure 10. The devices ranged in length from $70 \mu$ to $200 \mu$. Each device is from $1.5 \mu$ to $2 \mu$ thick. Table 3 (Appendix) shows a description of the dimensions for both clamp and gripper. Figure 7 illustrates the motion characteristics of the device. Small probes attached to piezo-electric actuators were used to operate the devices, see Figure 31 (Appendix). The micro-gripper end-handles closed when the connector bar is moved backwards, Figure 7 (Sec. 3.1). The micro-clamp separated when equal and opposite forces are applied on the handles, Figure 7 (Sec. 3.1). The clearance of the micro-devices is approximately $.85 \mu$.

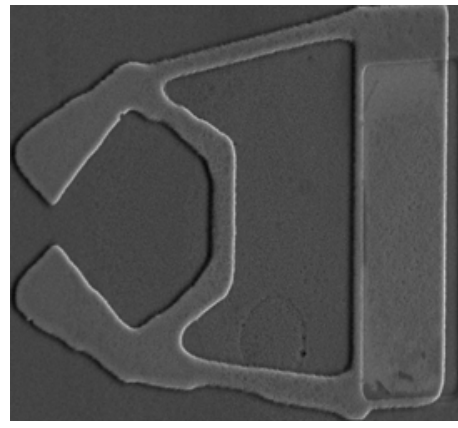

(a)

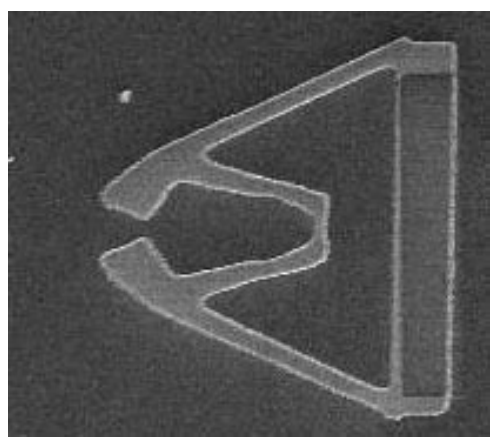

(b)

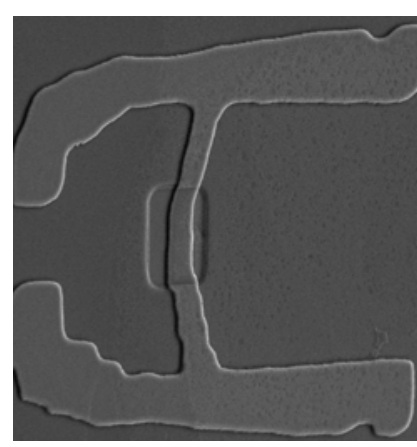

(c)

Figure 10: Fabricated compliant micro-gripper (a) \& (b) and micro-clamp (c)

\subsection{Solution Technique}

In this research, I have fabricated both the micro-clamp and the microgripper using the surface micro-machining process. These results can be compared to the information collected by Ananthesuresh (1995) bulk micro- 
machining process used to fabricate a compliant micro-gripper. In this endeavor the main objective was to illustrate the functional performance of the devices. Essentially, this was to show some movement of the devices in the prescribed direction under a set of different loading conditions. The test structures works such that if a voltage is applied to the piezo-electric actuator they move a probe attached to the mechanisms.

\subsubsection{Performance Criteria For Compliant Mechanism}

When designing compliant mechanisms, it is most important to establish a functional design that generates the desired output motion when subjected to certain input forces. Once this design is established, performance constraints can be imposed to optimize the device. In this thesis, I have considered four important criterions: (1) mechanical advantage-which is the force amplification,

(2) geometric advantage-which is motion amplification, and (3) stress free thinfilm mechanisms to limit buckling, and manufacturability.

\subsubsection{Computation of Mechanical and Geometric Advantage}

For a compliant mechanism to operate properly the design must have a degree of flexibility. Thus, for a given input, a desired output must be realized. Two terms are used to quantify this behavior, Mechanical Advantage (MA) and Geometric Advantage (GA). The Mechanical Advantage is the measurement of the force amplification. It is defined as the ratio of the output force $\left(F_{\text {out }}\right)$ to input force $\left(F_{\text {in }}\right)$. Consider the micro-gripper in figure 11 , there is a force applied $\left(F_{\text {in }}\right)$ to the center connector that causes the arms to move closer together. If a test sample is placed between the arms of the gripper there will be a force applied 
$\left(F_{\text {out }}\right)$ to the sample due to the contact between the gripper arms and the sample specimen. The Geometric Advantage (GA) is the measurement of the motion amplification. It is the ratio of output deflection ( $\left.d_{\text {out }}\right)$ to input deflection $\left(d_{\text {in }}\right)$. For this case, consider the compliant micro-clamp. Figure 11 illustrates the direction of the applied force. This applied force causes the clamp handles to move from position $\mathrm{X}_{1 \text { handles }}$ to $\mathrm{X}_{2 \text { handles. }}$. As the clamp handles move some distance $\Delta_{\text {handles, }}$, it causes the clamp arms to move some distance $\Delta_{\text {arms }}$.
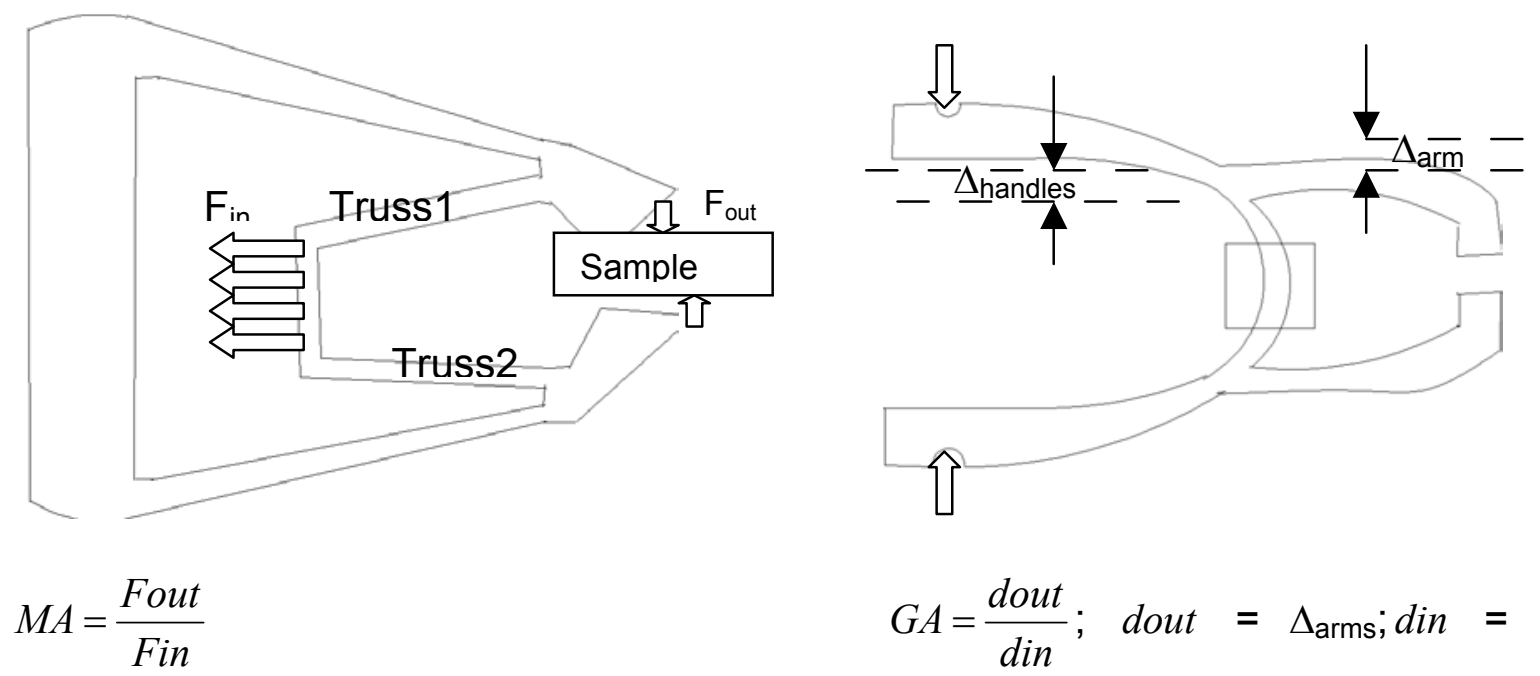

$\Delta$ handles

Figure 11: The Mechanical and Geometrical Advantage Illustration

In rigid body mechanisms that have a single input and single output port, the Geometric Advantage (GA) is equivalent to the inverse of the Mechanical Advantage (MA). However, this assumption doesn't hold for compliant mechanisms. The difference is that energy is not conserved between the input and output. For compliant devices energy is stored in the flexible components themselves. It is important to note that in rigid bodies $M A \bullet G A=1$; however, in compliant designs this value is $<1$. Thus, the goal in compliant mechanism 
designs is to get this value as close to 1 as possible. This will insure a high mechanical efficiency, which is the ratio of work output to work input.

This research provides an analysis of the MA of this devices as well as the GA. Using my testing configuration a voltage is applied to the piezoelectric probes. These probes supply the force in a desired direction, see Figure 7. Using the built-in scale of the microscope lens, we are able to determine the GA value for the design. The input deflection equals $1.5 \mu \mathrm{m}$ and the output deflection equals $2.8 \mu \mathrm{m}$ for applied voltage $20 \mathrm{~V}$. Based on the formulation, $G A=\frac{d o u t}{d i n}, \mathrm{GA}$ is equal to 1.83 . To determine the MA for the device, it is important to place a sample in the gripper arms. However, in this evaluation only an approximation of the MA values is determined. To approximate this value, we developed a freebody diagram. Consider Figure 11; in this diagram assume that $F_{\text {in }}$ is distributed evenly in Truss 1 and Truss2. Assume that the force applied $\mathrm{F}_{\text {in }}=40 \mu \mathrm{N}$, it can be determined that the force in Truss $1\left(F_{\text {truss } 1}\right)=20 \mu \mathrm{N}$. Consider only Truss 1 which is half of the complete gripper mechanism, I can calculate a force in the gripper arms equal to $8 \mu \mathrm{N}$. This would equal a total gripper arm force of $16 \mu \mathrm{N}$. Based on the formulation, $M A=\frac{\text { Fout }}{\text { Fin }}$, MA is equal to .40 . Assuming rigid body behavior $M A \bullet G A=1$, the value for this design is .74 , which is close to unity.

\subsubsection{Analysis of Stress in Thin-Film}

When fabricating MEMS devices each of the deposition and etching steps has a chance of introducing stress into the thin-film that can be detrimental to the device performance. 
In this case, too much stress in the polysilicon film could cause the devices to simply break and become unusable. Thus, the total stress in a thinfilm is defined as $\sigma_{\text {total }}=\sigma_{\text {ext }}+\sigma_{\text {th }}+\sigma_{\text {int }}$. The external stress, $\sigma_{\text {ext }}$, is typically due to another deposited layer on the current thin film. The thermal stress, $\sigma_{\text {th }}$, is due to the differences in the thermal coefficient between the two layers. This thermal stress can be defined using the following formula: $\sigma_{\text {th }}=$ $\frac{\text { Efilm }}{1-v f i l m} \int_{T_{o}}^{\text {Tdep }}(\alpha f i l m 1-\alpha f i l m 2) d T$, where Efilm is the Young's Modulus of the film, $v$ film is the poison ratio of the film, $\alpha f i l m 1$ and $\alpha f i l m 2$ are the average coefficient of thermal expansion for the deposited layers. Finally, the intrinsic stress, $\sigma_{\text {int, }}$ in the film is due to several variables including deposition temperature, ambient pressure, method of film preparation, and the type of substrate. There are many other variables not listed that effect intrinsic stress. Analyzing these stresses give us a range of acceptable levels in the devices.

In this analysis, the stress in the polyslicon layer is the most important. The polysilicon layer is the mechanical structure layer. The stress in this layer will determine if the device will be usable. It is determined that the initial stress in the polysilicon layer was 220 to 230 MPA. To decrease this stress, the wafers were annealed. This annealing process reduced the stress by $88 \%$.

In addition to the stress in the film, it is important to also consider the stress introduced by the external loading. The piezo-electric probes introduced this external loading, see figure 31 . Because we are considering elastic behavior, this structure should follow Hooke's Law. Hooke's Law states that the stress in a 
mechanism is directly proportional to the Elastic Modulus $(E)$ and the strain $(\varepsilon)$, i. e. $\sigma=E \varepsilon$. In this analysis a total stress computation should include not only the stress in the film, but also include the stress introduced by the external loading. The total stress can be written as $\sigma_{\text {total }}=27.5 \mathrm{MPA}+\mathrm{E} \varepsilon$, where $27.5 \mathrm{MPA}$ is the average stress of the annealed polysilicon layer and $E_{\varepsilon}$ is the stress due to the external loading of the mechanical structure.

\subsubsection{Manufacturability of the Devices}

This analysis illustrates the successful fabrication of a compliant micromechanism suspended on a polysilicon post. When considering manufacturability, the goal is to have a high yield ratio. Often the yield can be lower due to fluctuations in the fabrication process and design tolerances, too name a few. Note that section 2.5 describes the difference in designs and yields produced by both surface and bulk micromachining processes. In the research sampling, we fabricated 160 micro-grippers and 120 micro-clamps on 8 separate wafers, 4 wafers were dedicated to micro-grippers and the other 4 dedicated to micro-clamps. This provided a sample size of 640 micro-grippers and 480 microclamps. Table 5 below shows, a distribution of successfully device fabrication. From this table we can successfully predict the yield ratio.

Table 3: Profile of Compliant Micro-gripper and Micro-clamp Fabrication

\begin{tabular}{|l|c|c|c|c|}
\hline \multicolumn{1}{|c|}{ \# Of Samples } & WAFER 1 & WAFER 2 & WAFER 3 & WAFER 4 \\
\hline $\begin{array}{l}\text { \#Successful } \\
\text { Micro-gripper }\end{array}$ & 40 & 60 & 80 & 70 \\
\hline $\begin{array}{l}\text { \#Unsuccessful } \\
\text { Micro-gripper }\end{array}$ & 120 & 100 & 40 & 50 \\
\hline $\begin{array}{l}\text { \#Successful } \\
\text { Micro-clamp }\end{array}$ & 30 & 45 & 65 & 65 \\
\hline $\begin{array}{l}\text { \#Unsuccessful } \\
\text { Micro-clamp }\end{array}$ & 90 & 75 & 55 & 55 \\
\hline
\end{tabular}


From Table 3 it is shown that the fabrication process started to yield better results with wafer 3 and wafer 4 . Essential wafer 1 and wafer 2 were used to calibrate the process and determine suitable times for each of the processing steps, namely Reactive-lon Etch (RIE) and Buffered Oxide Etch (BOE) rates. As these processes became more refined, the yield ratio increased.

\subsection{FEA Analysis Von Mises Stress}

Figures 21 and 22 (micro-gripper) and Figures 29 and 30 (micro-clamp) show the von mises stress distribution and deflection plot of the mechanism under the prescribed $5 \mu$ loading. This is a simulated analysis of the expect behavior that the device should exhibit. It is show to illustrate the location of stress concentration areas. This model is a valid representation because the same laws that govern macro mechanisms performance also affect the microscale.

\subsection{Compliant MEMS Application}

Frecker, Kota, and Kikuchi (1998) proposed that there are inherent limitations to some topology optimization design problems. Most of the problems are evaluated in terms of prescribed input forces and output deflections relationships. When you want to use compliant devices in conjunction with smart materials this method doesn't satisfy all the variables, for example, this formulation does not consider issues such as impedance matching and minimization of elastic losses. However, smart devices can greatly benefit compliant mechanisms development in MEMS. Compliant mechanisms will allow for more design options and functionality. 


\subsection{Challenges}

Some of the greatest challenges were the result of using a wet etch process for the polysilicon layer. The first tested solution was undiluted $\mathrm{HNO}_{3}+$ $\mathrm{CH}_{3} \mathrm{COOH}+\mathrm{HF}$. This solution had an etch rate of $5 \mu / \mathrm{min}$. In this process, I used a $1.5 \mu-2.0 \mu$ layer, this results in an etch time of less than 1 minute. Unfortunately, the polysilicon layer was over etched and unusable. After a few more tests, I discovered that a 10 second etch time would give better results, but roughens the device surface. The final solution of diluting this solution with a $25: 1$ ratio of $\mathrm{H}_{2} \mathrm{O}(\mathrm{DI})$ to $\mathrm{HF}$ slowed down the etch process.

The idea process was to use the RIE (Reactive-ion etch) plasma of $\mathrm{CF}_{4}+$ $\mathrm{O}_{2}$ for the anisotropic etch. We were able to successfully use RIE to etch the polysilicon layer, see Polysilicon RIE etch recipe in the Appendix.

Note that when designing mechanisms, it is important to design with nearly equal device dimensions, especially if you choose to release the device with wet etching technique (i.e. BOE, etc). If the devices have great variations in dimensions, it is possible to over etch smaller features that should be maintained after the processing. However, it is possible to design some features a little too large. How much to oversize these features; can be determined by measuring the etchant rates of the materials that are used during subsequent processing steps and how these etchant effect material on any other layer. This will result in the components being etched down to the correct dimensions during the etching step. Figure 12 illustrates how the wet chemistry etching was starting to destroy the polysilicon devices. 

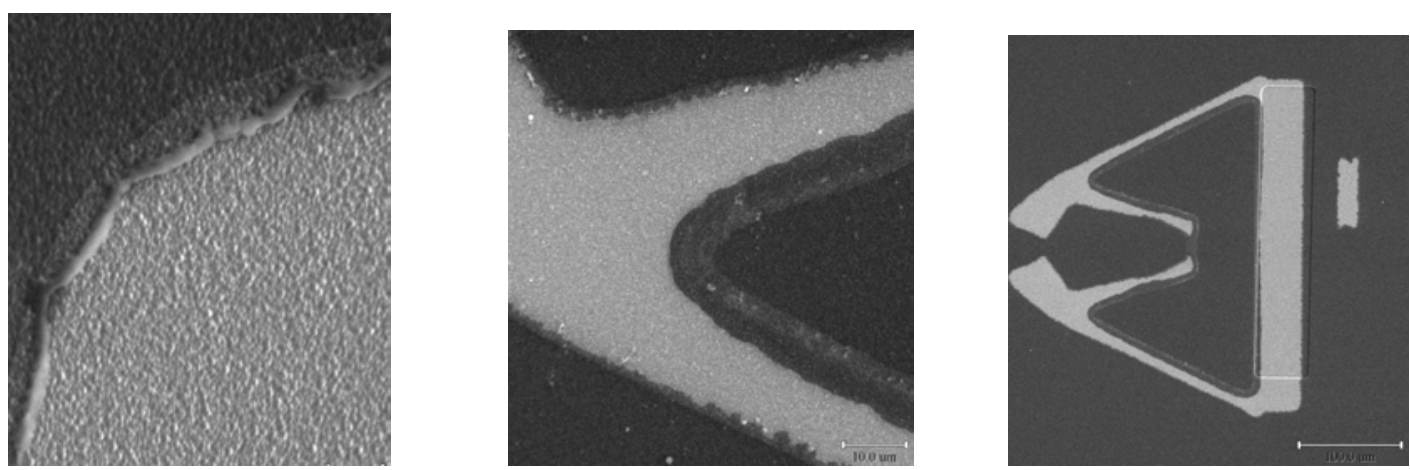

Figure 12: Wet-chemistry etching destroying polysilicon layer

While testing, the structure would break under the applied loading. This is probably due to the degradation of the polysilicon structure layer during subsequent processing steps. For both designs, it is very difficult to take accurate measurements due to the breakage. 


\section{CHAPTER 5. CONCLUSION AND DISCUSSION}

\subsection{Discussion of Results and Analysis}

In this research proposal, I have attempted to support surface micromachining as a valid process for the fabrication of compliant MEMS. This research has provided a step by step fabrication process as well as micrograph images of the fabricated device. It illustrates that a free-standing compliant mechanism can be successfully fabricated using surface micromachining. However, this research does not provide complete support for polysilicon as the structure layer for fully compliant devices. Each design had the tendency to break when the external loading is applied. Based on the calculated values for the mechanical and geometric advantage, it is deduced that $30 \%$ of the work input is stored in the device. Meaning that this work is not observed at the output. This led to the conclusion that the energy stored in the devices eventually caused the devices to break. In theory, the designs should exhibit flexibility and transform back into the original shape, unless the elastic limit is exceeded. This flexibility and transformability are major characteristics of compliant mechanisms; however, they are not observed in this investigation. This suggests that the design algorithms, fabrication process, or the structure material doesn't support proper device behavior. A simulation of the expected behavior and the stress distribution profile for each device is provided in the Appendix. A re-design to compensate for the high stress areas will improve the device actuation without device failure. This also concludes that compliant devices are somewhat inefficient as far as energy transfer. 


\subsection{Perspective for Future Research}

A proposed future endeavor for this research is to refine and improve the numerical optimization analysis to take into account microfabrication processes. This will allow the utilization of material properties to be a factor in optimizing the design for such devices. Another possible investigation is the use of other materials as the structure layer. Other polymers or a more flexible metal might yield better results when applying the external force. In addition to new materials. implementing electronics on the devices to automate the actuation can illustrate the operating device more easily. 


\section{APPENDIX}

Reactive Ion Polysilicon Etch Recipe $\quad 48$

$\begin{array}{ll}\text { Definitions and Terms } & 49\end{array}$

Figure 13: L-EDIT Illustration on Micro-Compliant Gripper 53

Figure 14: L-EDIT Illustration on Micro-Compliant Clamp 53

Figure 15: Design Domain for Compliant Gripper 54

Figure 16: FEM Deflection Mesh of Compliant Gripper 54

Figure 17: Optimized 1/2 Gripper Configuration 54

Figure 18: Gripper Configuration no. 2 no. 4

Figure 19: Gripper Configuration no. 3 no. 5

Table 4: Dimensions of Micro-Gripper Design 55

Figure 20: Micro-gripper Fabrication Schematic 56

Figure 21: Compliant Micro-Gripper Stress Distribution 57

Figure 22: Compliant Micro-Gripper Deflection 57

Figure 23: Design Domain for the Compliant Clamp 58

Figure 24: FEM Deflection Mesh if Compliant Clamp 58

Figure 25: Optimized $1 \frac{1}{2}$ Clamp Configuration 58

Figure 26: Clamp Configuration no. 1 no. 2

Figure 27: Clamp Configuration no. 3 no. 4

Table 5: Dimensions of Micro-Clamp Design 59

$\begin{array}{ll}\text { Figure 28: Micro-clamp Fabrication Schematic } & 60\end{array}$

Figure 29: Stress Distribution of Deformed Clamp 61

Figure 30: Schematic of deflected and non-deflected Device 61 
Figure 31: Test Structure For Device Characterization 


\section{Reactive Ion Polysilicon Etch Recipe}

Machine: 700 Series Waf'r/Batch: Plasma Processing System.

\section{Initial Step \#00}

Main Process Pump: Turbo

Initial Pump Down: Turbo

Base Pressure: $\quad 8.0 \times 10-5$

Hold After Base: $\quad$ No

\section{Pumpdown Step \#01}

Pump Configuration: Turbo

Base Pressure: $\quad 8.0 \times 10-6$

Hold After Base: $\quad$ Yes

Hold Time: $\quad$ 5:00 (min)

\section{Process Step \#02}

Gas $\mathrm{CH} 1-1$ (O2): 4 (sccm)

Gas CH1-3 (CF4): 46 (sccm)

Pressure: $\quad 090$ (mtorr)

RF Generator: None

Termination Time: $\quad 00: 45(\mathrm{sec})$

\section{Process Step \#03}

\begin{tabular}{ll}
\hline Gas CH1-1 (O2): & 4 (sccm) \\
Gas CH1-3 (CF4): & 46 (sccm) \\
Pressure: & 090 (mtorr) \\
RF Generator: & RF1 \\
RF Conf: & RIE \\
RF Control: & DCV \\
RF1 Setpoint: & 300 (volts) \\
RF1 Max: & 450 (volts) \\
Termination Time: & $15: 00$ (min)
\end{tabular}

\section{Pumpdown Step \#04}

Pump Conf: Turbo

Base Pressure: $\quad 8.0 \times 10-5$

Hold: No

\section{End Sequence}

Gas Line Cleanup:

Final Pumpdown

None

Hold After Base

Turbo

Hold Time:

Yes

Vent:

5:00 (min)

No 


\section{DEFINITIONS}

\section{Biomimetics}

Scientific research using biological systems to develop synthetic systems with the desired functionality.

\section{Bulk Micromachining}

Microfabrication technique in which the desired features are carved out of a bulk piece of material using reagents such as acids or bases. A protective resistant layers masks the areas that are to be left untouched.

\section{Boron Diffusion}

The second stage in the silicon wafer processing. After all the planar contours of the mechanical components have been patterned, this technique is applied. The depth of diffusion determines the thickness of the components.

\section{BICMOS}

Bipolar complementary metal-oxide-semiconductor

\section{CMOS}

Complementary metal-oxide-semiconductor. This is a process used to fabricate VLSI (very large scale integration) devices. These devices consist of layers of conductors, insulators, and semiconductors on a silicon wafer.

\section{Compliant Mechanisms}

Mechanisms that exhibit motion and force transmission properties by elastic deformation.

\section{Chemical Vapor Deposition}


Process used to deposit layers of material such as silicon oxide, silicon nitride onto the silicon wafers.

\section{Cyclic Mechanical Failure}

Failure and fatigue due to alternative loading.

\section{Geometric Advantage}

A non-intrinsic characteristic of a mechanism determined as the ratio of a deflection output to a deflection input.

\section{Homogenization Method}

A method to obtain optimal designs for structures with known space and loading conditions. This is a mathematical theory for obtaining average elastic moduli of material with periodic microstructures.

\section{Informatics}

The study of using computer and statistical techniques to the management of information.

\section{LIGA}

Deep-etch synchrotron lithography and high precision replication processes. This is an acronym for Lithographie Galvanoformung Abformung. This process allows the fabrication of microstructures of any lateral shape with structural heights.

\section{Mechanical Advantage}

A non-intrinsic characteristic of a mechanism determined as the ratio of a force output to a force applied. 


\section{Metrology}

Science dealing with measurements.

\section{Photolithography}

The process used to transfer the designs from the photomask onto the wafer.

\section{Photoresist}

Material that undergoes a chemical reaction when exposed to light.

\section{Photomask}

The created by exposing, or writing the required pattern in a resist layer spun on top of a chromium layer.

\section{Recessing Silicon}

The process of etching away the oxide layers everywhere there is a projection.

\section{Reticles}

Refers to a patterned substrate that contains one to about six copies of the chip pattern and is used with an optical projection system to image the pattern onto the wafer.

\section{Surface Micromachining}

Microfabrication technique in which the desired features is formed by selective deposition and removal of material in alternating layers.

\section{Topology}

The way to define the geometry of a component. This is essentially the shape and the size. 


\section{Tribology}

The science of the mechanisms of friction, lubrication, and wear of interacting surfaces that are in relative motion 


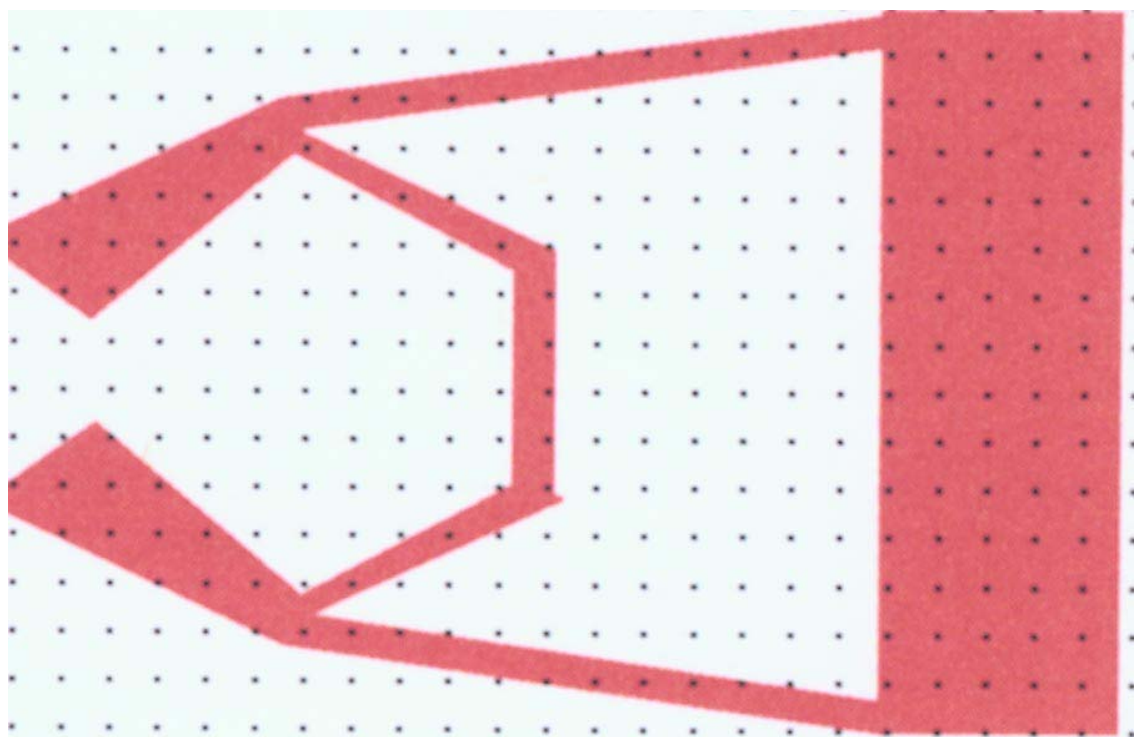

Figure 13: L-EDIT Illustration on Micro-Compliant Gripper

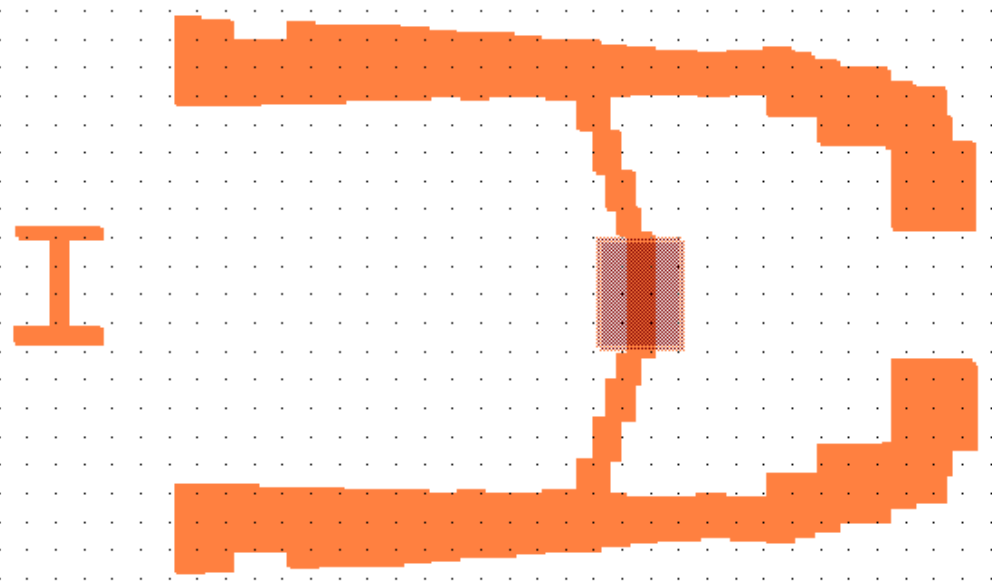

Figure 14: L-EDIT on Micro-Compliant Clamp 


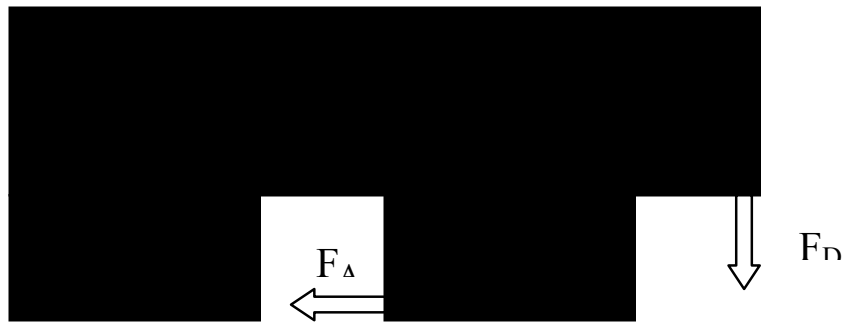

Figure 15: Design Domain for the Compliant Gripper

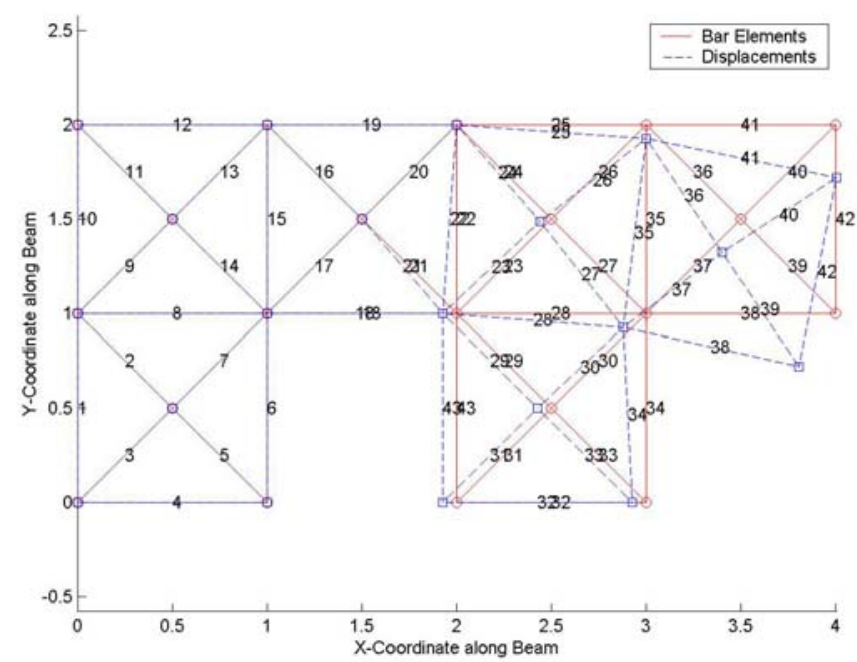

Figure 16: FEM Deflection Mesh of Compliant Gripper

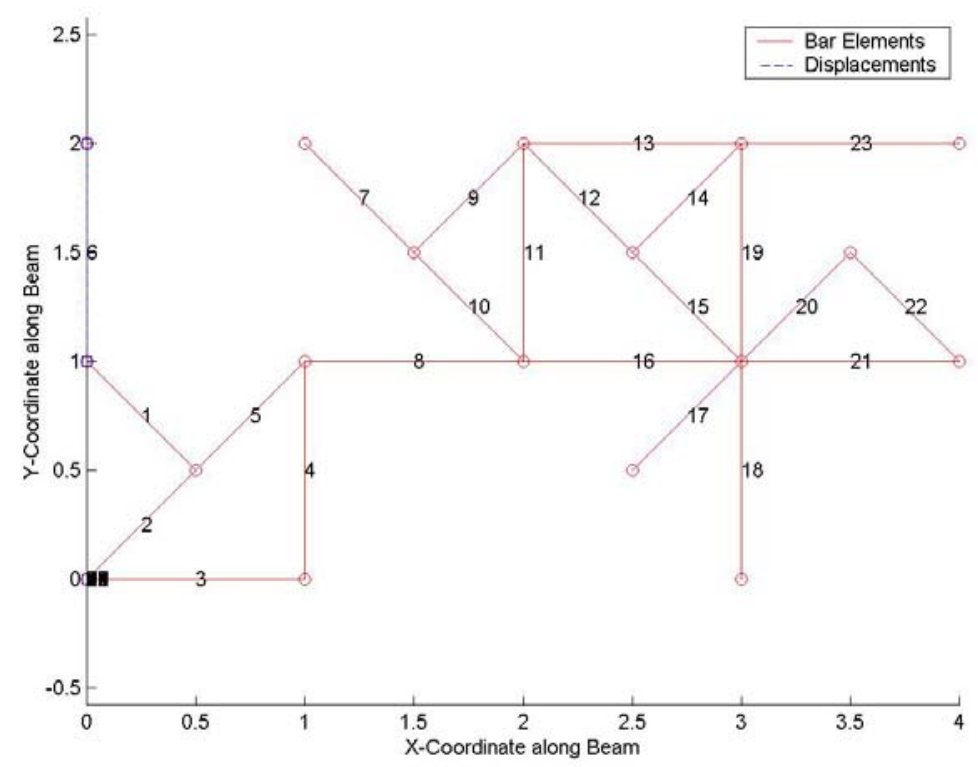

Figure 17: Optimized 1/2 Gripper Configuration Structural Interpretation 


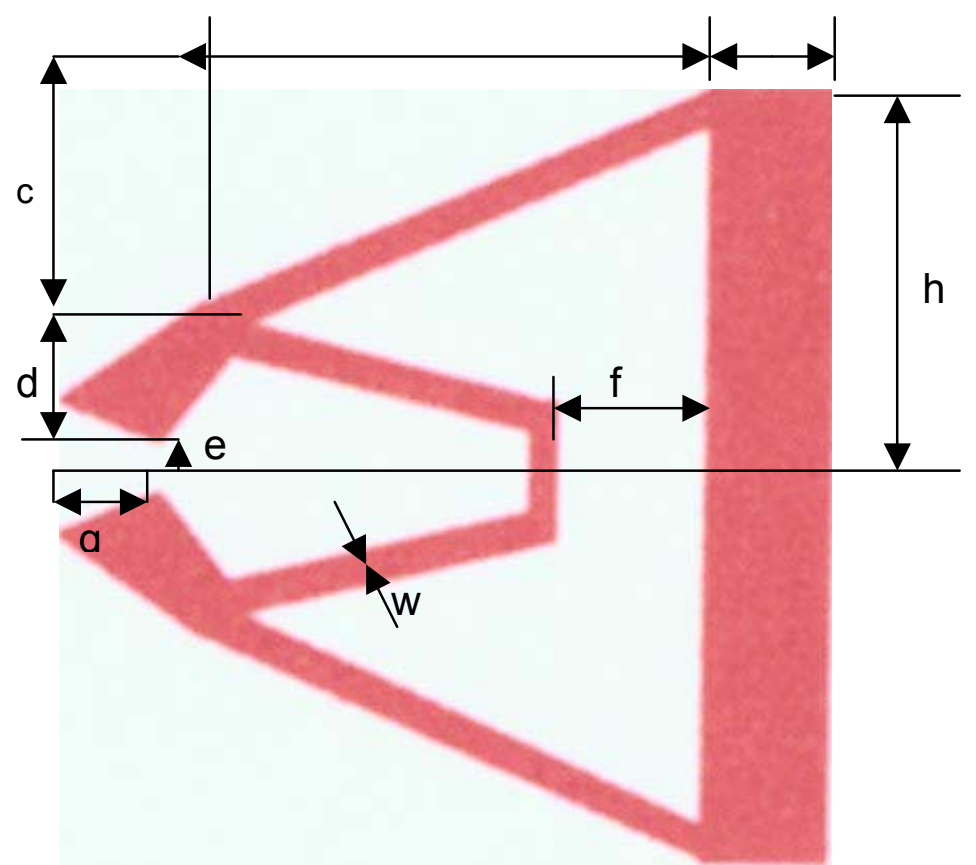

Figure 18: Gripper Configurations no. 2 no. 4

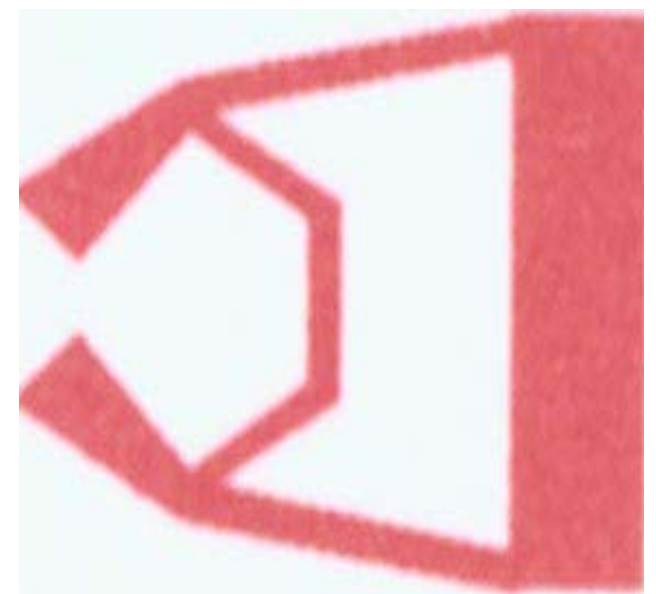

Figure 19: Gripper Configuration no. 1 no. 3

Note $\mathrm{t}=$ thickness

Table 4: Dimensions of Micro-Gripper Design

\begin{tabular}{|c|c|c|c|c|c|c|c|c|c|c|}
\hline No. & $\mathrm{a}$ & $\mathrm{b}$ & $\mathrm{c}$ & $\mathrm{d}$ & $\mathrm{e}$ & $\mathrm{f}$ & $\mathrm{g}$ & $\mathrm{h}$ & $\mathrm{w}$ & $\mathrm{t}$ \\
\hline 1 & 50 & 150 & 25 & 40 & 10 & 65 & 60 & 65 & 5 & 1.5 \\
\hline 2 & 50 & 200 & 80 & 95 & 15 & 65 & 65 & 130 & 5 & 1.5 \\
\hline 3 & 50 & 150 & 25 & 40 & 10 & 65 & 60 & 65 & 5 & 2.0 \\
\hline 4 & 50 & 200 & 80 & 95 & 15 & 65 & 65 & 130 & 5 & 2.0 \\
\hline
\end{tabular}




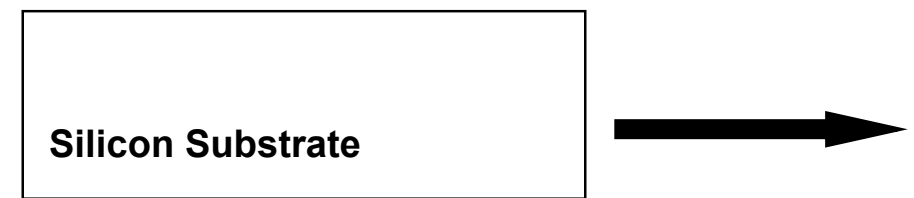

1. Initial Silicon Wafer layer grow

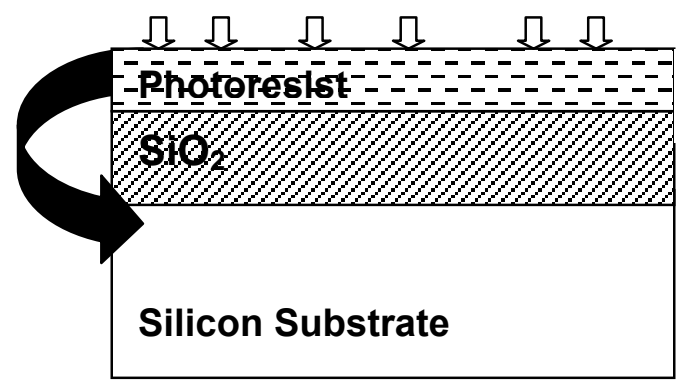

3. Expose to UV Radiation material

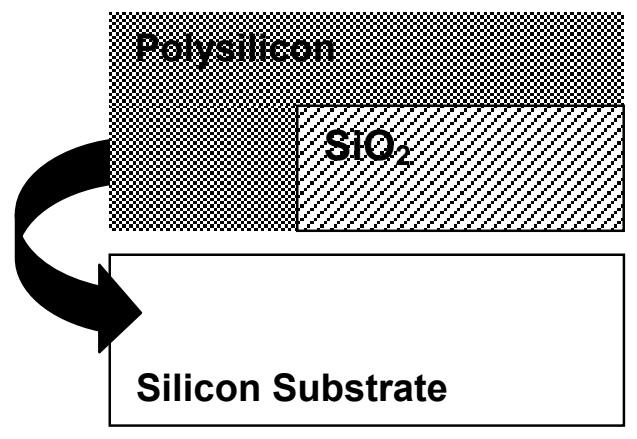

5. Polysilicon Depostion release device

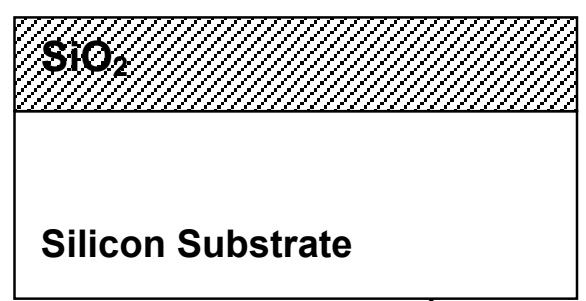

2. . $85 \mu \mathrm{m}$ oxide

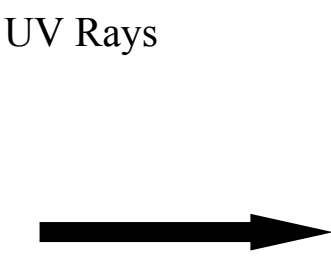

Silicon Substrate

4. Remove exposed

Strip photoresist.

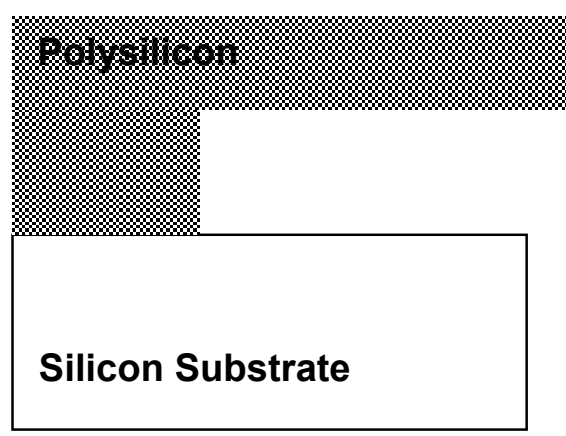

6. Remove $\mathrm{SiO}_{2}$ to

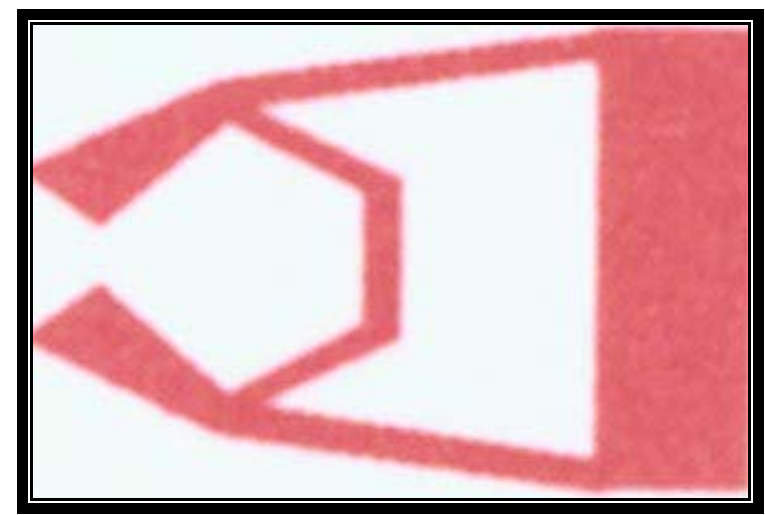

Top-View of fabricated micro-gripper 
Figure 20: Micro-gripper Fabrication Schematic

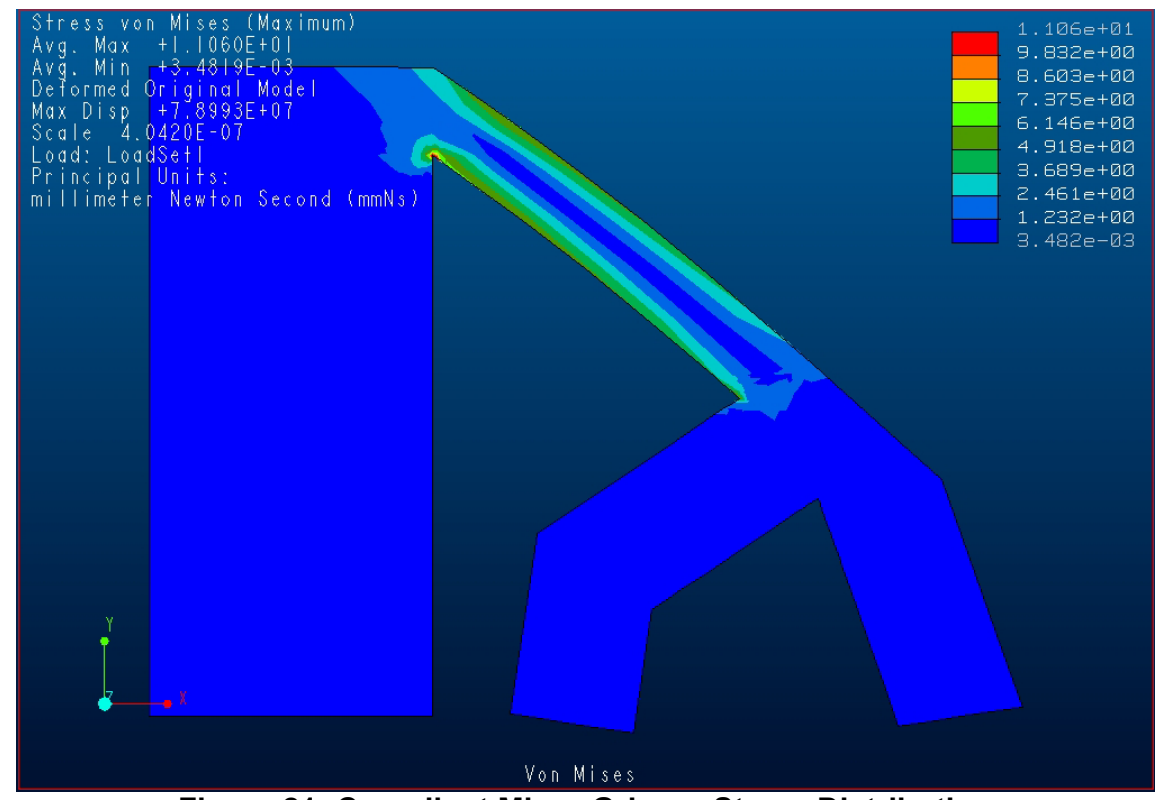

Figure 21: Compliant Micro-Gripper Stress Distribution

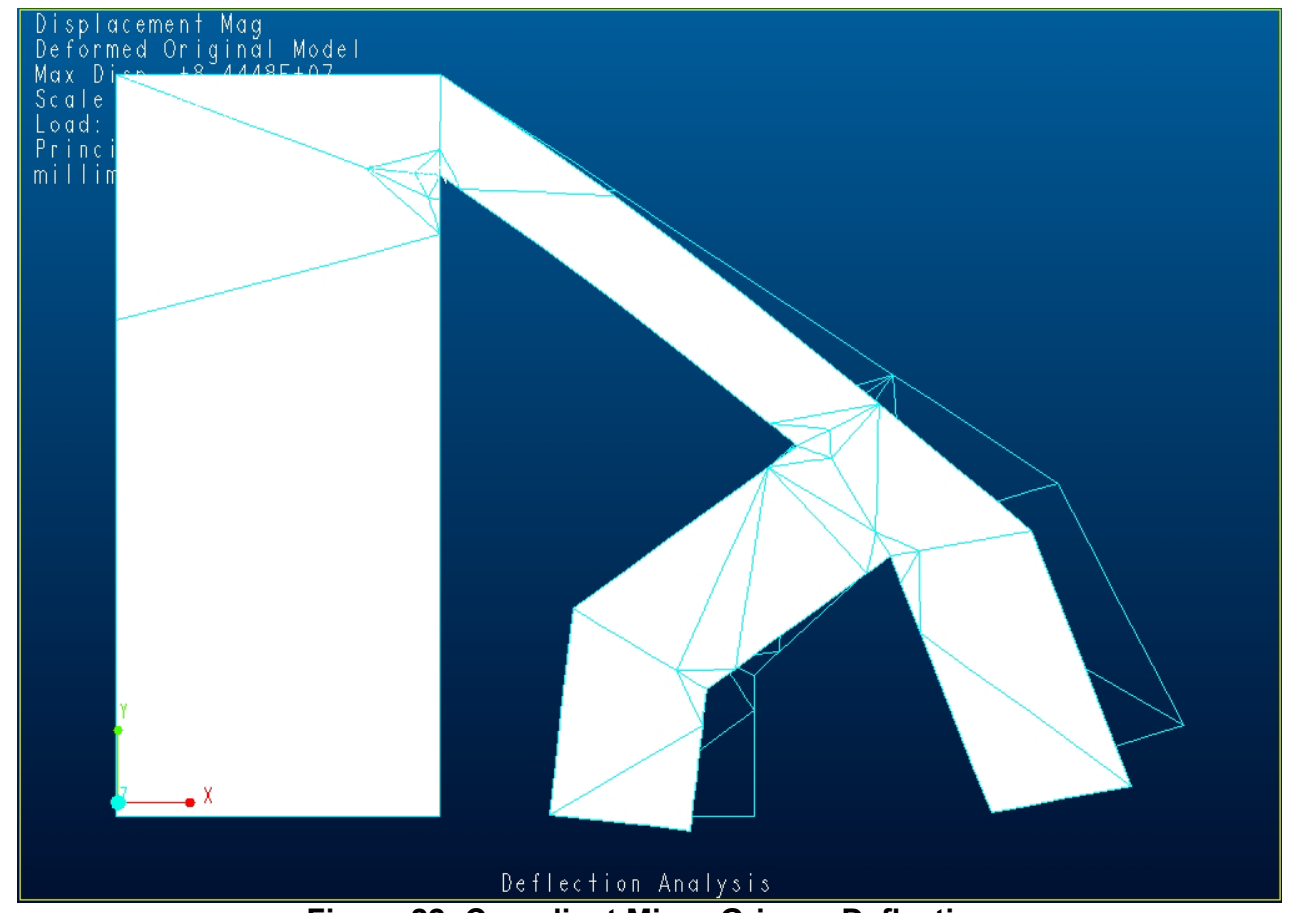

Figure 22: Compliant Micro-Gripper Deflection 


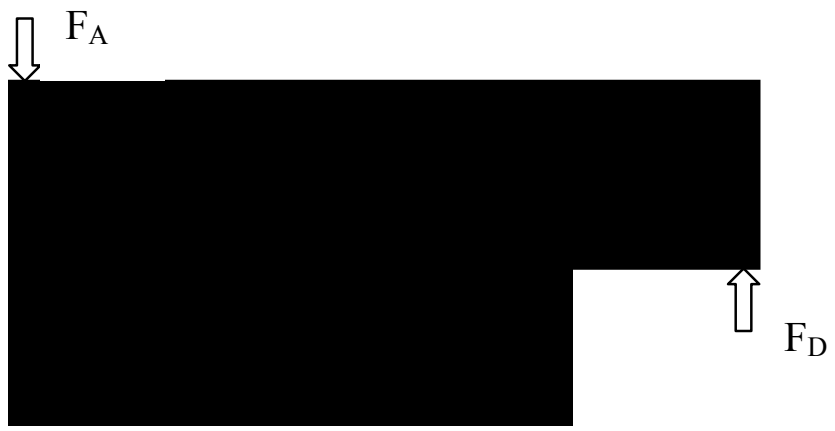

Figure 23: Design Domain for the Compliant Clamp

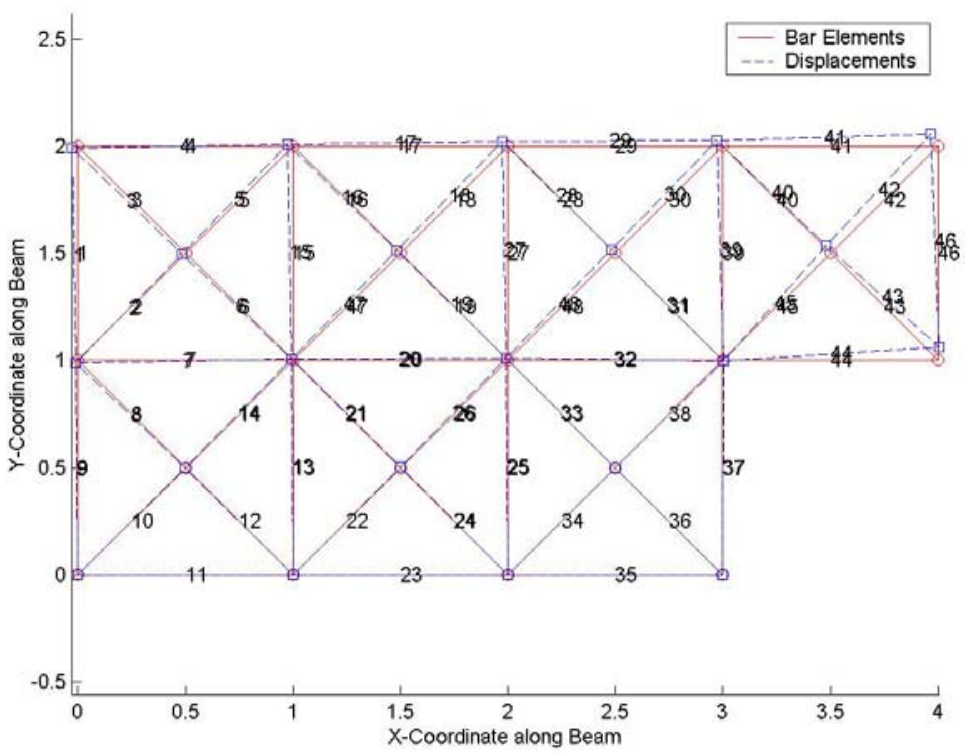

Figure 24: FEM Deflection Mesh of Compliant Micro-clamp

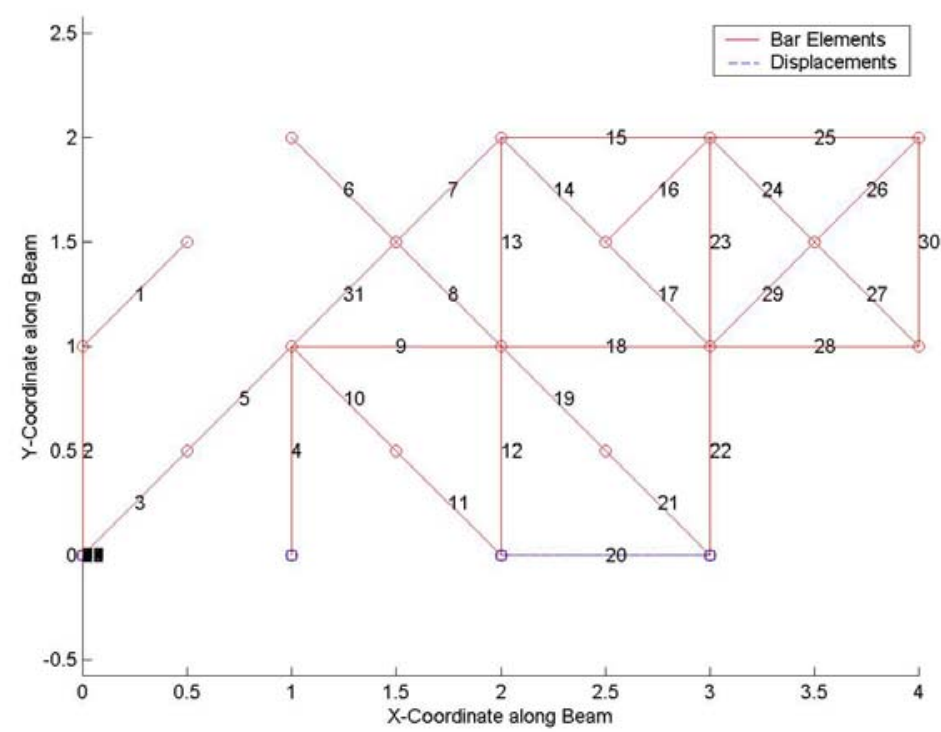

Figure 25: Optimized 1/2 Micro-clamp Structural Interpretation 


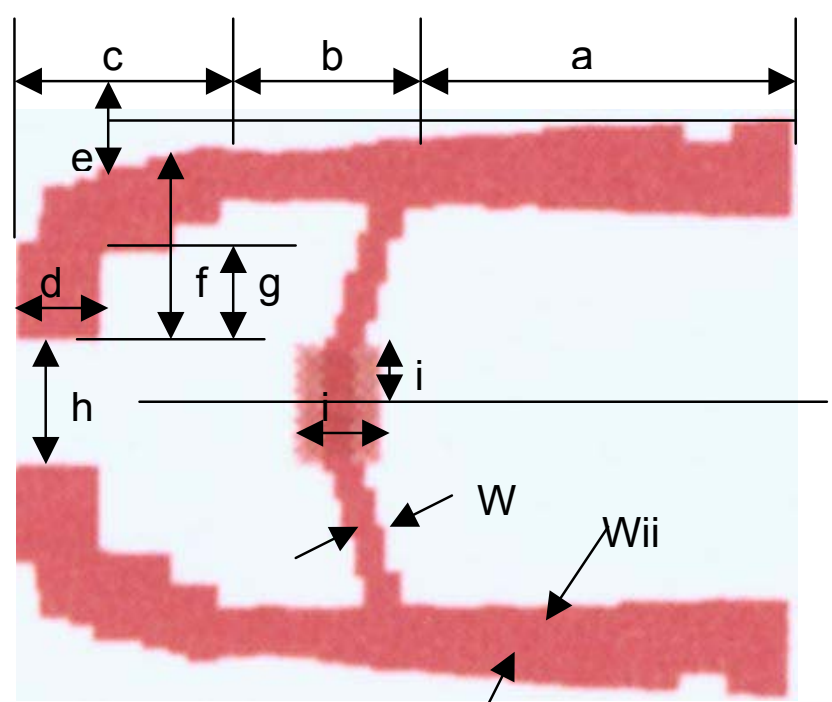

Figure 26: Clamp Configurations no.1 no. 2

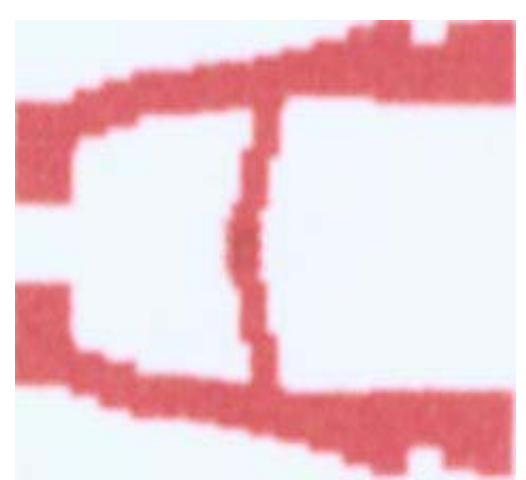

Figure 27: Clamp Configurations no.3 no. 4

NOTE: $\mathrm{t}=$ thickness

Table 5: Dimensions of Micro-Clamp Design

\begin{tabular}{|c|c|c|c|c|c|c|c|c|c|c|c|c|c|}
\hline No. & $\mathrm{a}$ & $\mathrm{B}$ & $\mathrm{C}$ & $\mathrm{d}$ & $\mathrm{e}$ & $\mathrm{f}$ & $\mathrm{g}$ & $\mathrm{h}$ & $\mathrm{i}$ & $\mathrm{j}$ & wi & wii & $\mathrm{t}$ \\
\hline 1 & 140 & 40 & 100 & 30 & 15 & 30 & 30 & 50 & 20 & 25 & 5 & 10 & 1.5 \\
\hline 2 & 140 & 40 & 100 & 30 & 15 & 30 & 30 & 50 & 20 & 25 & 5 & 10 & 2.0 \\
\hline 3 & 70 & 20 & 50 & 15 & 10 & 15 & 15 & 25 & 10 & 15 & 7 & 15 & 1.5 \\
\hline 4 & 70 & 20 & 50 & 15 & 10 & 15 & 15 & 25 & 10 & 15 & 7 & 15 & 2.0 \\
\hline
\end{tabular}




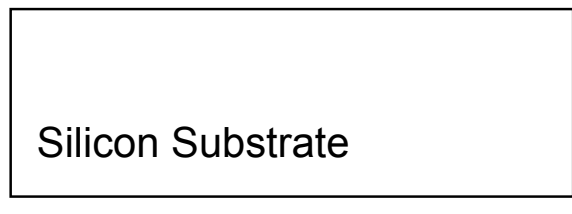

1. Initial Silicon Wafer

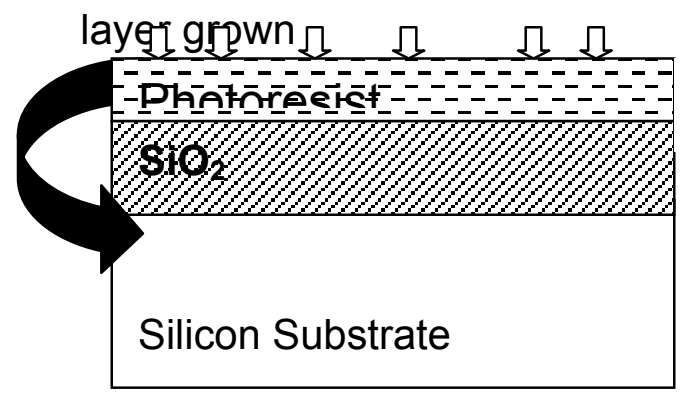

3. Expose to UV Radiation

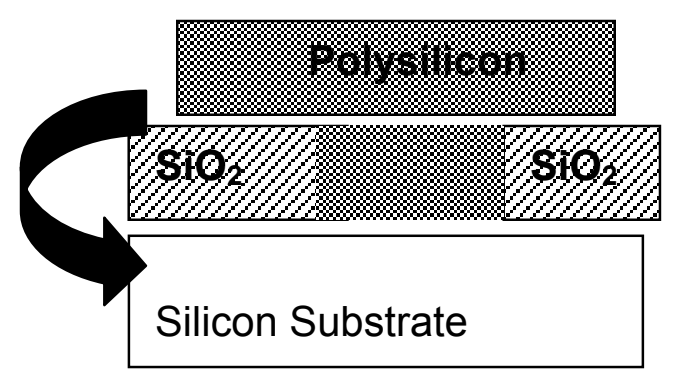

5. Polysilicon Depostion release device

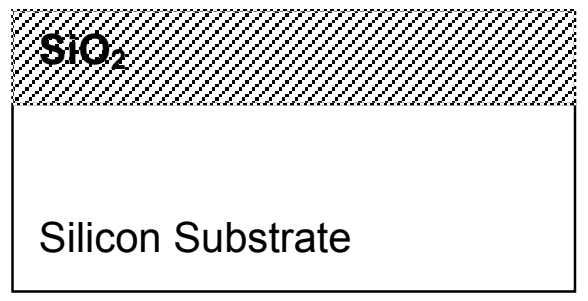

2. .85 $\mu \mathrm{m}$ oxide

\section{UV Rays}

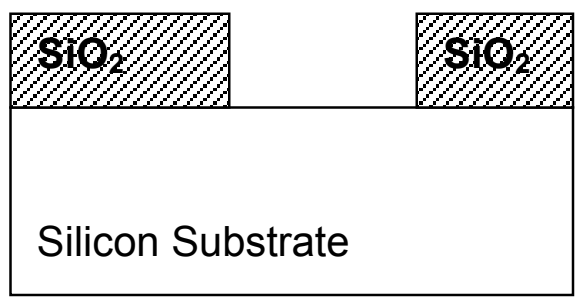

4. Remove exposed resist Strip remaining resist

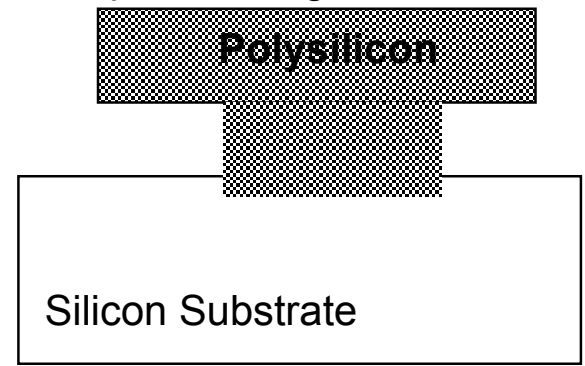

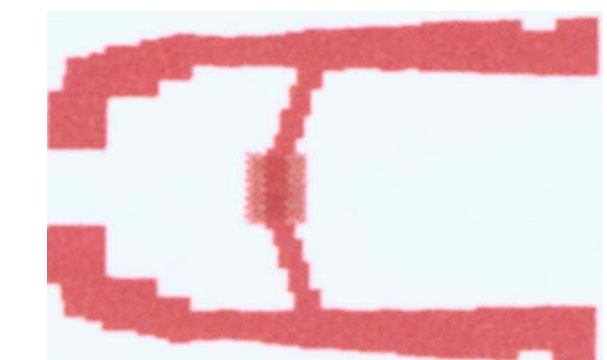

Top-View of fabricated micro-clamp

Figure 28: Micro-clamp Fabrication Schematic 


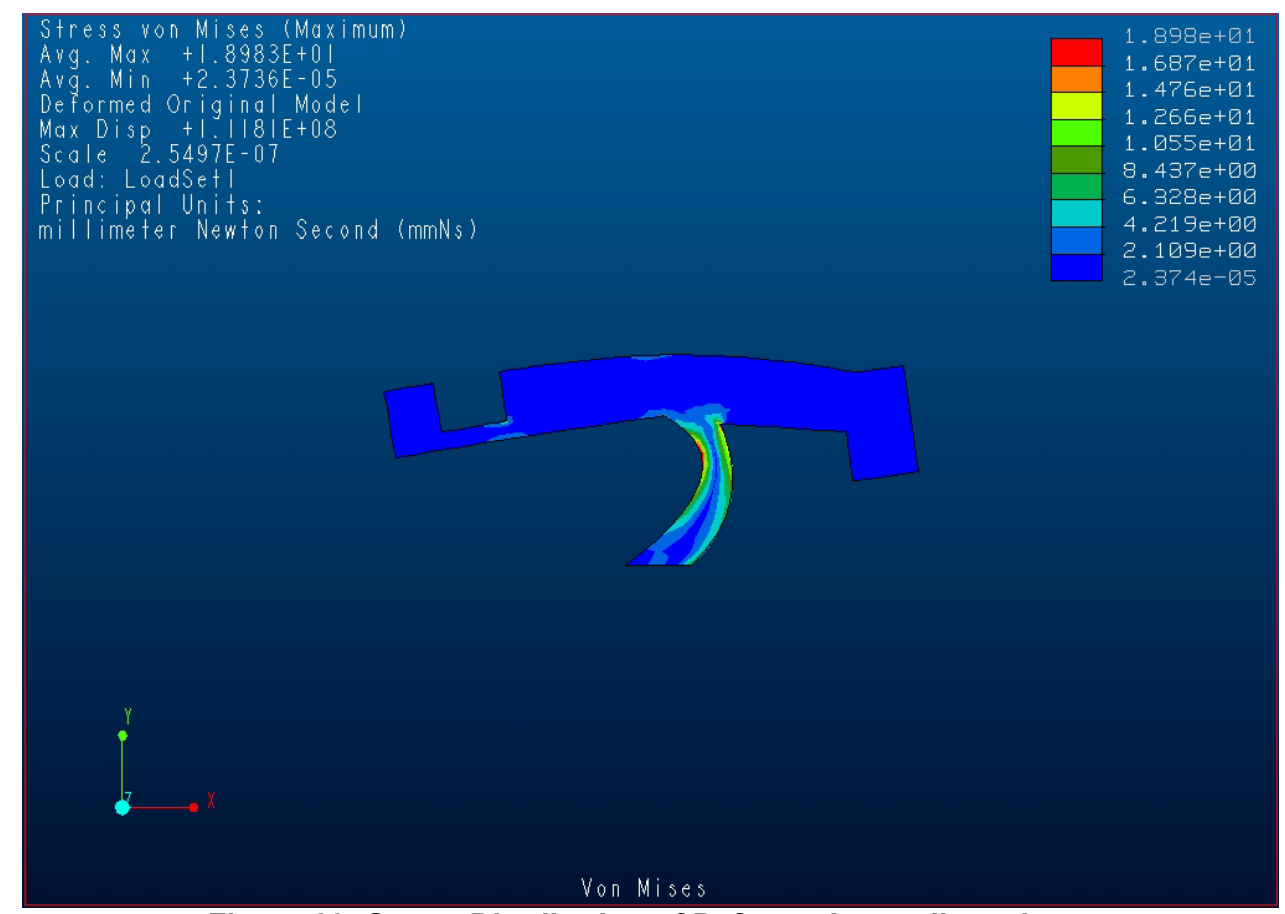

Figure 29: Stress Distribution of Deformed compliant clamp

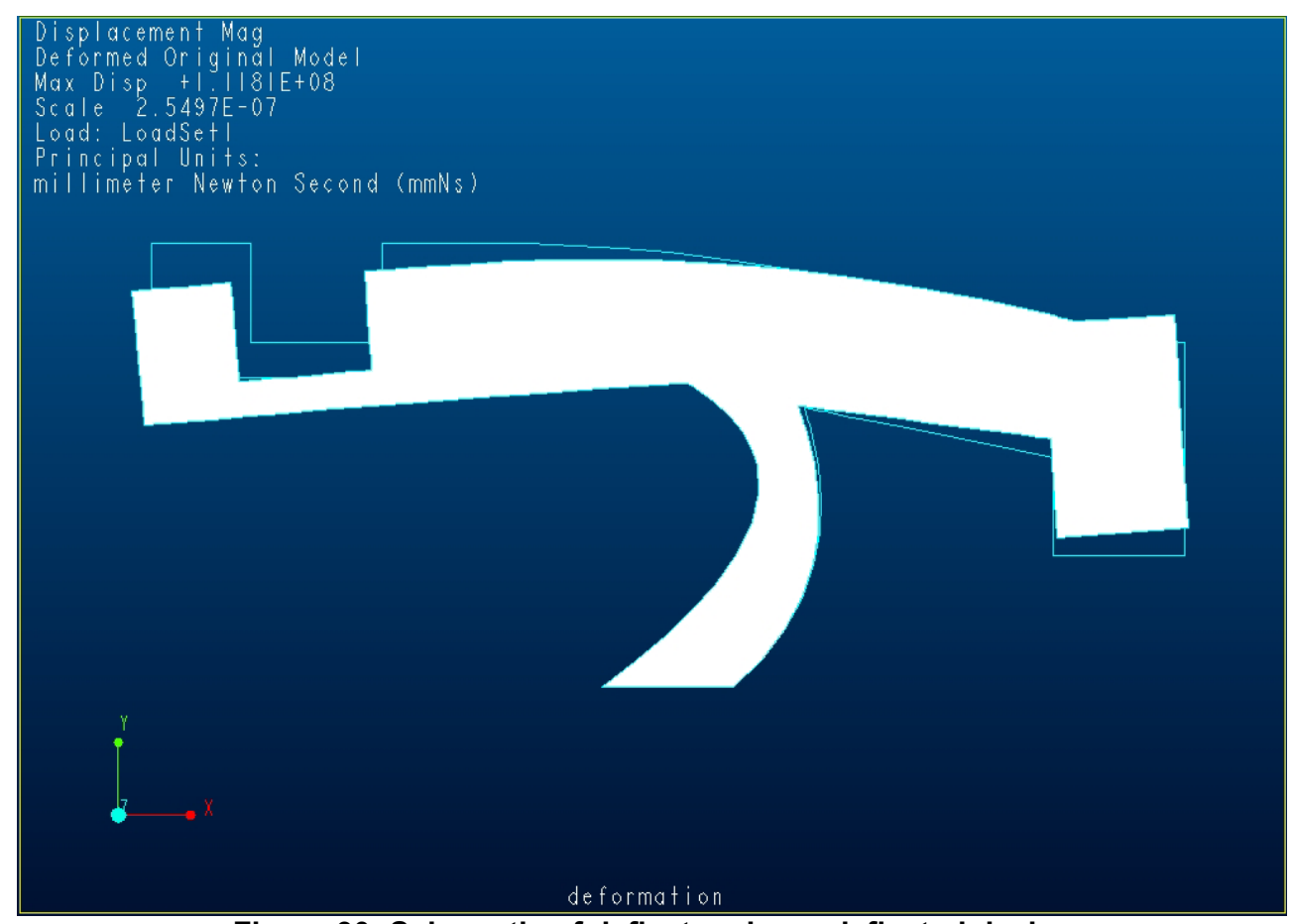

Figure 30: Schematic of deflect and non-deflected device 


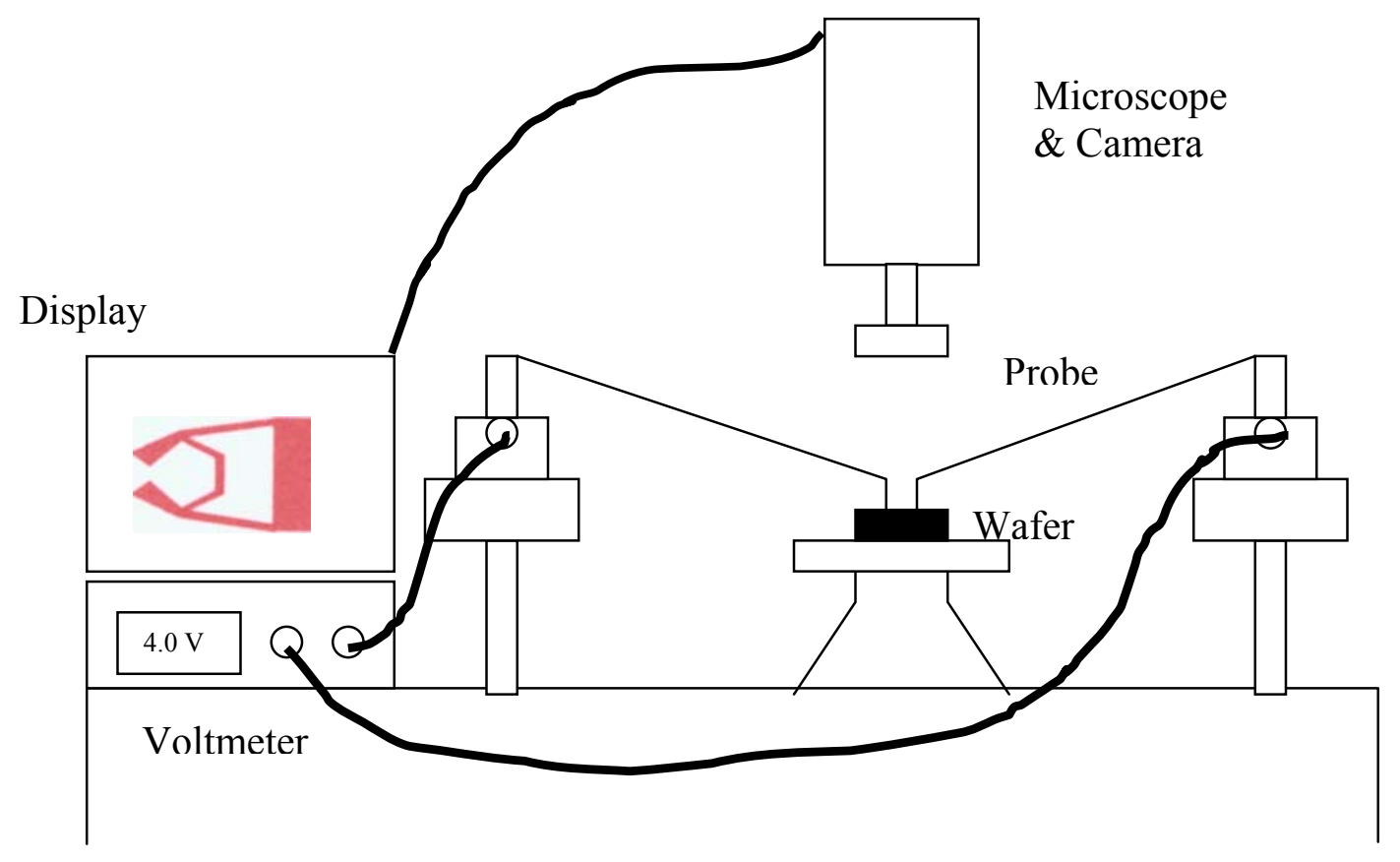

Figure 31: Test Structure For Device Characterization 


\section{REFERENCES}

Ananthasuresh, G. K., "A New Design Paradigm for Micro-Electro-Mechanical Systems \& Investigation on the Compliant Mechanism Synthesis," Doctoral Dissertation, University of Michigan, Ann Arbor, Michigan, 1994.

Ananthasuresh, G. K., Kota, S., and Gianchandani, Y., 1994a "A Methodical Approach to the Synthesis of Micro Compliant Mechanism". Technical Digest, Solid-State Sensor and Actuator Workshop, June 13-16, 1994, Hilton Head Island, South Carolina, pp. 189-192.

Ananthasuresh, G.K., Kota, S., and Kikuchi, N., 1994b. "Strategies for Systematic Synthesis of Compliant MEMS". Proceedings 1994 ASME Winter Annual Meeting, Chicago, Illinois, November, 1994, DSC-Vol. 55-2.

Bao, M., Wang, W., "Future of Microelectromechanical Systems," Sensors and Actuators, 1996, pp. 135-141.

Bendsoe, M. P., and Kikuchi, N., 1988. "Generating Optimal Topologies in Structural Design Using Homogenization Method". Computer Methods in Applied Mechanics and Engineering, 71 (1988), pp. 197-224.

Boronkay, T. G., Mei, C., "Analysis and Design of Multiple Input Flexible Link Mechanism," Journal of Mechanisms, Vol. 5 No.1, 1970, pp. 29-49.

Burgess, S. C., Moore, D. F., Edwards, K., Shibaike, N., Klaubert, H., Chiang, $\mathrm{H}-\mathrm{S}$, "The Design of a Microelectromechanical Accelerometer, ${ }^{\text {st }}$ International Workshop on Collaborative Design, Tokyo, January 1995.

Burgess, S. C., Moore, D. F., Edwards, K., Shibaike, N., Klaubert, H., "A Study of Mechanical Configuration Optimisation in Micro-system," Research in Engineering and Design, 1997, pp. 46-59.

Burns, R.H., and Crossley, F.R.E., 1968, "Kinetostatic Synthesis of Flexible Link Mechanism," ASME Paper No.68-Mech-36.

Bustillo, J. M., Howe, R. T., Muller, R. S., "Surface Micromachining for Microelectromechanical Systems," Proceedings of IEEE, Vol. 86, No.8, August 1998, pp. 1552-1569.

Byers, F. K., Midha, A., "Design of A Compliant Gripper Mechanism," Proceedings of the $2^{\text {nd }}$ Applied Mechanisms and Robotics Conference, 1991, pp. XC.1-1-XC.1-12. 
Chen, L. Y., Zhang, Z. L., Yao, J. J., Thomas, D. C., MacDonald, N. C., "Selective Chemical Vapor Deposition of Tungsten For Microdynamic Structures," Sensors and Actuators, 1989, pp. 82-87.

Erdman, A. G., Sandor, G. N., Mechanism Design Analysis and Synthesis, Vol 1, 1993.

Fan, L. S., Tai, Y. C., and Muller, R. S., 1987, "Pin Joints, Gears, Springs, Cranks, and other Novel Micromechanical Structures," Transducers '87, pp. 849-856.

Frecker, M., Ananthasuresh, G. K., Nishiwaki, S., Kikuchi, N., and Kota, S., 1997, "Topological Synthesis of Compliant Mechanisms Using Multi-Criteria Optimization". Journal of Mechanical Design, Transaction of the ASME, Vol 119, No. 2 June 1997, pp. 238-245.

Frecker, M., Kota, S., Kikuchi, N., 1998. "Optimal Design of Compliant Mechanisms for Smart Structures Applications", Mathematical and Control in Smart Structures, SPIE Conference. San Diego, California, March 1998 pp. 234-240.

French, P. J., Gennissen, P. M., Sarro, P. M., "New Silicon Micromachining Techniques for Microsystems," Sensors and Actuator, 1997, pp. 652-662.

French, P. J., Sarro, P. M., "Surface versus Bulk Micromachining: The Contest for Suitable Application," Micromechanics and Microengineering, 1998, pp. 45-53.

Fujita, H., "Microactuators and Micromachines," Proceedings of IEEE, Vol. 86, No. 8, Aug. 1998, pp. 1721-1732.

Fujita, H., and Gabriel, K. J., 1991, "New Opportunities for Micro Actuators," Digest of the Technical Papers, 1991 International Conference on Solid-State Sensors and Actuators, San Francisco, CA, June 23-27, pp. 14-20.

Gianchandani, Y. B., Najafi, K., "A Bulk Silicon Dissolved Wafer Process for Microelectromechanical Devices", Journal of Microelectromechanical Systems, Vol 1, No.2, 1992, pp. 77-85.

Han, Jung-In, Han, Chul-Hi, "A Self-Aligned Offset Polysilicon Thin-Film Transistor Using Photoresist Reflow", IEEE Electron Device Letters, Vol. 20, No.9, Sept. 1999, pp. 476-477.

Her, I., 1986, "Methodology for Compliant Mechanism Design," Ph.D Dissertation, Purdue University, West Lafayette, IN. 
Her, I. and Midha, A., 1987, "A Compliance Number Concept for Compliant Mechanisms and Type Synthesis," ASME Journal of Mechanisms, Transmissions and Automation in Design, Vol. 109, No.3, pp. 348-355.

Hetrick, J. A., Kikuchi, N., Kota, S., "Size and Shape Optimization of Compliant Mechanisms: An Efficiency Formulation," Proceedings of DETC'98, 1998, Sept. 13-16, pp. 1-7.

Hetrick, J. A., Kikuchi, N., Sridhar, K., "Robustness of Compliant Mechanism Topology Optimization Formulation," Conference on Mathematics and Control, Mar. 1999, pp. 244-254.

Howell, L. L., and Midha, A., 1994a. "A Method for the Design of Compliant Mechanisms with Small-Length Flexure Pivots". Journal of Mechanical Design, Transaction of the ASME, Vol 116, March, 1994, pp. 281-291.

Howell, L. L., and Midha, A., 1994b. "A Generalized Loop Closure Theory for Analysis and Synthesis of Compliant Mechanisms". Proceedings 1994 ASME Design Engineering Technical Conferences, Machine Elements and Machines Dynamics, DE-Vol. 71, Minneapolis, Minnesota, Sept. pp. 12-14, 1994.

Jensen, B. D., Howell, L. L., Gunyan, D. B., Salmon, L. G., "The Design and Analysis of Compliant MEMS Using The Pseudo-Rigid Body Model", Journal of Microelectromechanical Systems, ASME 1997, pp. 119-126.

Kiefer, D. S., "Reactive Ion Etch Recipes For Failure Analysis", Motorola Plasma Applications, Motorola Corporation, Chandler, AZ., 1997.

Kim, C. J., Pisano, A. P., Muller, R. S., and Lim, M. G., "Design, Fabrication, and Testing of Polysilicon Gripper," ASME Winter Meeting, Dallas, Texas, Nov.1990, pp. 99-109.

Kovacs, G. T., Maluf, N. I., Petersen, K. E., "Bulk Micromachining of Silicon," Proceedings IEEE, Vol. 86, No.8, Aug. 1998, pp. 1536-1548.

MATLAB, 2000, MATLAB: The Language of Technical Computing, The Mathworks Inc., Natick, MA.

Mehregany, M., Gabriel, K. J., Trimmer, W. S., "Selective Chemical Vapor Deposition of Tungsten for Microdynamics Structures," Proceedings of IEEE Microelectromechanical Systems Workshop, Feb. 1989, pp. 82-87.

MEMS Exchange, "What is MEMS Technology?" Retrieved [August 5, 1999] from the [MEMS Exchange] database on the World Wide Web: http://www.mems-exchange.org/MEMS/what-is.htm. 
Mettlach, G. A., Midha, A., "Graphical Synthesis Techniques Toward Designing Compliant Mechanism," Proceedings of the $4^{\text {th }}$ National Applied Mechanisms \& Robotic Conferences, Vol. 2, 1996, pp. 61-01-61-10.

Multi-User MEMS Processes (MUMPs) Design Handbook, MCNC, Raleigh, NC. 1999, pp. 1-8.

Murphy, M. D., Howell, L. L., Midha, A., "Methodology For The Design of Compliant Mechanisms Employing Type Synthesis Techniques with Examples," Mechanism Synthesis and Analysis, ASME, DE-Vol. 70, 1994, pp. 61-66.

Nishiwaki, S., Frecker, M. I., Min, S., Kikuchi, N., "Topology Optimization of Compliant Mechanisms Using The Homogenization Method," International Journal For Numerical Methods in Engineering Vol. 42, 1998, pp. 535-559.

Norton, T. W., "On the Nomenclature and Classification and Mobility of Compliant Mechanisms," Master's Thesis, 1991, Purdue University, West Layfette, IN.

Paros, J., Weisbord, L., "How to Design Flexure Hinges," Machine Design, Nov. 1965, pp. 151-156.

Petersen, K. E., "Silicon as a Mechanical Material," Proceedings of IEEE, Vol. 70, No.5, May 1982, pp. 420-457.

Rai-Choudhury, P., Handbook of Microlithography, Micromachining, and Microfabrication, Vol. 1-2, 1997, Bellingham, WA.

Ramesham, R., Ghaffarian, R., Kim, N.P., "Reliability Issues of COTS MEMS for Aerospace Application," Proceedings of the Jet Propulsion Laboratory, Sept. 1999, pp. 53-63.

Saggere, L., Kota, S., "Synthesis of Distrbuted Compliant Mechanisms for Adaptive Structures Application: An Elasto-Kinematic Approach", Proceedings 1997 ASME Design Engineering Technical Conferences, Sept. 14-17, 1997, DETC97/DAC-3861.

Salamon, B. A., "Mechanical Advantage Aspects in Compliant Mechanisms Design," Master's Thesis, 1989, Purdue University, West Lafayette, IN.

Salamon, B. A., Midha, A., "A Methodology for Compliant Mechanisms Design: Part 1 - Introduction and Large-Deflection Analysis," Advances in Design Automation, Vol. 2, DE-Vol. 44-2, 1992, pp. 29-38. 
Salamon, L., Midha, A., "An Introduction to Mechanical Advantage in Compliant Mechanism," Advances in Design Automation, DE-Vol 44-2, $18^{\text {th }}$ ASME Design Automation Conference, 1992, Sept. 13-16, pp. 47-51.

Sarma, R, "Matlab Coding for Topology Optimization”, Unpublished 1997.

Save, M., and Prager, W., 1985, "Chapter 2: Problem Formulation and Optimality Criteria," Structural Optimization, Vol 1., Plenum Press, New York, pp. 37-39.

Saxena, A., Ananthasuresh, G. K., "Topological Synthesis of Compliant Mechanisms Using the Optimality Criteria Method," Proceedings of the DETC'98, ASME Design, Engineering Technical Conference, 1998, Sept 1316, pp. 1-11.

Sevak, N. M., and McLarnan, C. W., "Optimal Synthesis of Flexible Link Mechanisms with Large Static Deflections," ASME Design, 1974, No.74-DET83.

Shibaike, N., "Design of Micromechanisms Focusing on Configurations, Materials, and Processes. Journal of Material and Design, 1995, pp. 83-90.

Shoup, T. E., McLarnan, C. W., "A Survey of Flexible Link Mechanisms Having Lower Pairs," Journal of Mechanisms, Vol.6, No.3, 1971, pp. 97-105.

Spearing, S. M., "Materials Issues in Microelectromechanical Systems (MEMS)", Elsevier Sciences, July 1999, pp. 180-196.

Tuttle, S. B., "Chapter: 8, 1967, Semifixed Flexural Mechanisms," Mechanisms for Engineering Design, John Wiley and Sons, Inc., New York.

Winter, S. J., and Shoup, T. E., 1972, "The Displacement Analysis of Path Generating Flexible Mechanisms," Mechanisms and Machine Theory, Vol 7 , No.4, pp. 443-451. 


\section{ACKNOWLEDGEMENTS}

I wish to express thanks to my major professors, Dr. Radha Sarma and Dr. James Bernard. They both allowed me to create this research project and investigation. I would like to extend special thanks to Dr. Gary Tuttle for providing support and a research facility. I also thank Dr. George Jackson and Dr. Ted Okiishi for their support as well. I would like to say thank you to Curtis Sell, Tony Witt, and Allen Landin for their help at the Microelectronics Research Center at lowa State University. I thank my wife, Angela and my parents for their encouragement.

Finally, I give thanks to God for the wisdom and patience to complete this project.

This work was performed at Ames Laboratory under Contract No. W7405-Eng-82 with the U.S. Department of Energy and a National Science Foundation grant. The United States government has assigned the DOE report number IS-T 2178 to this thesis. 
